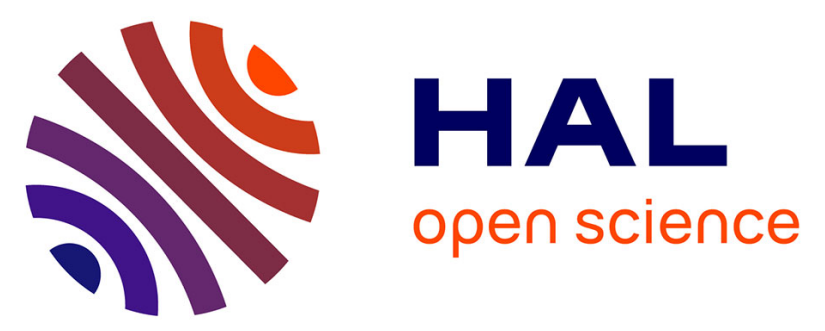

\title{
Dispersion control of raw and modified silica particles in PMMA. Impact on mechanical properties, from experiments to modelling
}

A. Siot, Romain Léger, C. Longuet, B. Otazaghine, A. S. Caro-Bretelle, N. Azema

\section{To cite this version:}

A. Siot, Romain Léger, C. Longuet, B. Otazaghine, A. S. Caro-Bretelle, et al.. Dispersion control of raw and modified silica particles in PMMA. Impact on mechanical properties, from experiments to modelling. Composites Part B: Engineering, 2019, 157, pp.163 - 172. 10.1016/j.compositesb.2018.08.104 . hal-01908915

\section{HAL Id: hal-01908915 https://hal.science/hal-01908915}

Submitted on 30 Oct 2018

HAL is a multi-disciplinary open access archive for the deposit and dissemination of scientific research documents, whether they are published or not. The documents may come from teaching and research institutions in France or abroad, or from public or private research centers.
L'archive ouverte pluridisciplinaire HAL, est destinée au dépôt et à la diffusion de documents scientifiques de niveau recherche, publiés ou non, émanant des établissements d'enseignement et de recherche français ou étrangers, des laboratoires publics ou privés. 


\title{
Dispersion control of raw and modified silica particles in PMMA. Impact on mechanical properties, from experiments to modelling
}

\author{
A. Siot, R. Léger, C. Longuet, B. Otazaghine, A.S. Caro-Bretelle*, N. Azéma
}

C2MA, IMT Mines Ales, Univ Montpellier, Ales, France

\begin{abstract}
*Corresponding authors: Anne-Sophie Caro-Bretelle
E-mail: anne-sophie.caro@mines-ales.fr
\end{abstract}

This paper has been first published online in Composites Part B in August $27^{\text {th }}, 2018$ (https://doi.org/10.1016/j.compositesb.2018.08.104).

\begin{abstract}
Structure and properties of PMMA/silica composites were characterized by various methods including SEM, TGA, rheology, microscopic level evaluation of nanoreinforcement effect on properties. Static modulus and yield strength of PMMA are enhanced with silica incorporation regardless of their dispersion or functionalization. However, composites ultimate properties are strongly affected by fillers characteristics. As expected, better performances are associated to well-dispersed and functionalized silica.

A finite element modelling with interface debonding through cohesive model and interfacial area is introduced to reproduce the sensibility of tensile test response to fillers surface functionalization. Experimental results confirmed this modelling: at constant low weight rate, ultimate composites properties are mostly affected by any microstructural changes. An analysis of local stress confirms the premature breakage of composite with pristine silica. Finally, by considering the expected properties, functionalization of silica is not always a necessity. Instead of particle functionalization, a dispersion protocol could be advantageously used to reach interesting composite properties.
\end{abstract}

Keywords: Dispersion, Silica functionalization, PMMA/Silica nanocomposites, Mechanical properties, Finite element modelling 


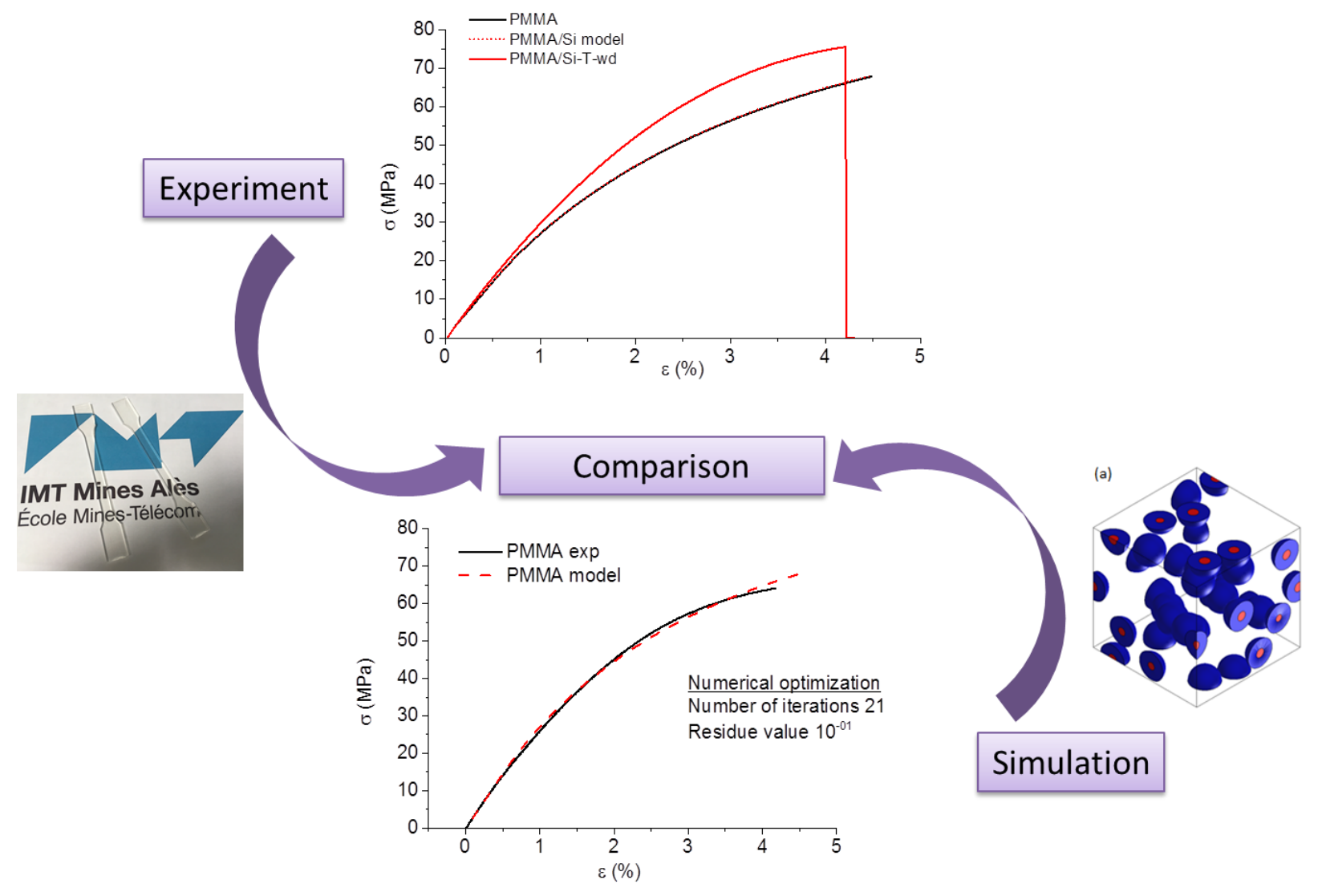

Graphical abstract 


\section{Introduction}

The use of nanoparticles as reinforcements for polymer matrices is relatively common due to their ability to impart strong improvements of performances at very low volume fractions in comparison to micrometric particles [1]. Nanocomposites find applications in various fields as their enhanced properties can be mechanical, electrical, optical...[2]. Particularly; nanoparticles are widely used to provide mechanical reinforcement to polymer matrices.

Numerous studies deal with elaboration and evaluation of mechanical properties of nanocomposites but, most of the time, only macroscopic properties are evaluated. At a macroscopic scale, it appears that incorporation of nanoparticles into a polymeric matrix influences elastic modulus but the influence of nanoparticles on local reinforcement mechanisms has hardly been studied [3]-[6]. However, micromechanisms of deformation of each phases, decohesion between matrix and fillers or cavitation phenomena inside fillers produce inhomogeneity of deformation and thus are responsible of microvoids creation and crazing [7].

Several studies showed the existence of an interphase in the vicinity of the nanoparticle in a polymer matrix [8]-[10]. It is proved that the interphase region can extend widely in hundreds nanometers around the particles [11]-[15]. This phenomenon could explain why a low amount of nanoparticles have a huge impact on composites properties. In fact, several studies showed that incorporation of a small weight percent in matrix modifies bulk polymer properties [16], [17]. It seems that a molecular dynamic change at the fillers proximity is observed due to the interactions between polymer chains and particles which explains the local change of properties in this interphase [18]-[20]. More precisely, the nature of the interphase between the mineral filler and polymer matrix depends of their interactions. They can interact by weak interactions like van der Waals strengths, $\mathrm{H}$-bond or electrostatic forces; or the filler/matrix system can be bound by strong chemical bonds (covalent or ionic-covalent). Particle surface modification is one of the most used strategy to change this interphase properties (and therefore affinity between fillers and matrix), and thus improve mechanical properties of nanocomposites [21].

Nowadays, several options are available to characterize interphase properties. Nanoindentation is used to determine the interphase size [22]. Atomic Force 
Microscopy (AFM) can be used to evaluate mechanical properties (AM-FM mode) [23], [24] of this region. However, these techniques are difficult to perform. The first difficulty lies in the preparation of samples. In fact, surfaces have to be perfectly realized to carry out AFM measurements which require an important know-how. Moreover, following the studied polymer, the choice of the tip is important. For example, in the PMMA case a not adapted sharp tip could break the molecular chains during measurements [25].

Modelling can be an indirect useful method to characterize this interphase in polymer composites. So far, nanocomposite structure can be simply regarded as a three phase multi-inclusions based on the continuum model. To extend their applicability to nanocomposites, analytical models were improved to consider coated inclusions [10], [15], [19], [26]-[28].

From the literature overview, it may be concluded that there is still a need for a simple method to identify microscopic phenomenon in order to predict mechanical behavior of composites filled with particles. The present study focuses on the mechanical behavior at macroscopic and microscopic scales of composites reinforced by silica particles at low volume fractions.

The goal of this work was to model the mechanical properties of PMMA/silica composites considering the existence of an interphase and several dispersion states. Composites were prepared with two different protocols with raw and surface modified silica particles leading to various microstructures. Silica particles were grafted with MMA oligomers to improve affinity with PMMA matrix. The particles used in this study were voluntarily chosen submicronic (i.e lower than micron but higher than 100nm), in order to obtain a "nano-effect" while keeping the observation of their dispersion state and of the particle/polymer interface easy. Finally, this study focused on the interest of silica particles functionalization instead of improvement of their dispersion by mechanical treatments. Functionalization has the advantages to improve the affinity between matrix and fillers, nevertheless, this strategy can be an expensive technique to develop.

\section{Materials and experiments}

\subsection{Materials under study}


PMMA Altuglass V825T was supplied by Arkema $\left(M_{w}=93,000 \mathrm{~g} / \mathrm{mol}\right.$, density=1.19) and used as received.

Silica spherical particles (density=2.64) were synthetized via a modified Stöber method. The specific surface area (BET) is $15.0 \pm 2.3 \mathrm{~m}^{2} / \mathrm{g}$ and the elementary particle diameter size is $D=470 \mathrm{~nm}$ with a narrow particle size distribution (supporting information).

Those synthetized silica particles were modified thanks to a grafting agent, obtained by free radical telomerization of methyl methacrylate (MMA), PMMA chains were then anchored onto the silica particles surface.

\subsubsection{Synthesis of the PMMA grafting agent}

This grafting agent corresponding to PMMA chains bearing one trialkoxysilane groups was obtained by free radical telomerization of MMA in presence of 3(mercaptopropyl)triethoxysilane (ETS). Into a $50 \mathrm{~mL}$ flask fitted with a condenser, $5 \mathrm{~g}$ $\left(5 \times 10^{-2} \mathrm{~mol}\right)$ of MMA, $0.48 \mathrm{~g}\left(2.0 \times 10^{-3} \mathrm{~mol}\right)$ of ETS, $0.08 \mathrm{~g}\left(4.9 \times 10^{-4} \mathrm{~mol}\right)$ of AIBN and $20 \mathrm{~g}$ of acetonitrile were introduced. Argon was bubbled through the mixture for $15 \mathrm{~min}$. The mixture was then stirred and heated at $60^{\circ} \mathrm{C}$ for 15 hours. After reaction, the polymer ETS-PMMA was purified by precipitation in methanol. A schematic representation of the grafting agent synthesis process is described in Figure 1.a.

\subsubsection{Synthesis of silica particles}

Silica particles $(470 \mathrm{~nm})$ were synthesized by a modified Stöber method already described in previous works [29], [30]. A schematic representation of the silica particle synthesis process is described in Figure 1.b. The synthesis reactions were performed in a $100 \mathrm{~mL}$ glass reactor. The synthesis used $26 \mathrm{~g}$ ammonia solution 3 $(25 \%)$ and $36 \mathrm{~g}$ deionized water. Then, they are injected into $212 \mathrm{~g}$ of pure ethanol $(\mathrm{EtOH}) .20 \mathrm{~g}$ TEOS is next added to the vial, while stirring at $50{ }^{\circ} \mathrm{C}$ for $10 \mathrm{~h}$ in view of the growth of nano-silica. Numerous studies have shown that all the process conditions like the temperature, TEOS ratio or process time for example have an important impact on the particles size [31]-[33]. Also, higher concentration of water resulted in larger size of the particle. 
At the end of this synthesis, ethanol was removed and replaced by water using evaporation under vacuum. Then, silica spheres were dried and purified, in order to remove remaining reactants, by washing and centrifugation methods.

\subsubsection{Silica functionalization}

Into a $100 \mathrm{~mL}$ flask fitted with a condenser, $1.5 \mathrm{~g}$ of silica, $0.15 \mathrm{~g}$ of ETS-PMMA and $50 \mathrm{ml}$ of toluene solution were introduced. The mixture was then stirred and heated at solvent reflux for $15 \mathrm{~h}$. The mixture was next centrifuged (speed: $5000 \mathrm{rpm}$ ) to eliminate the liquid phase and washed three times with THF. Finally, the obtained Silica-g-PMMA was dried under vacuum. A schematic representation of the grafting process is described in Figure 1.c.

(a) Synthesis of ETS-PMMA
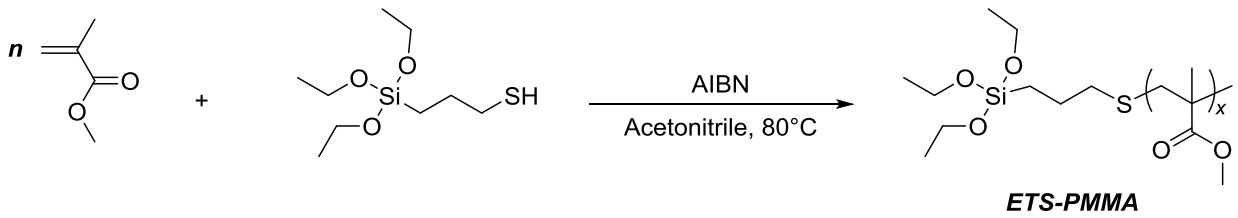

(b) Synthesis of silica particles

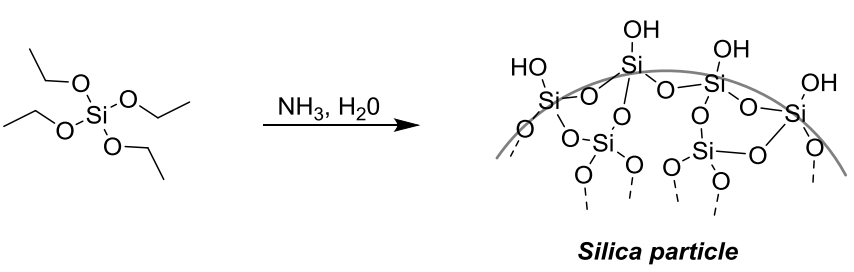

(c) Grafting of silica particles

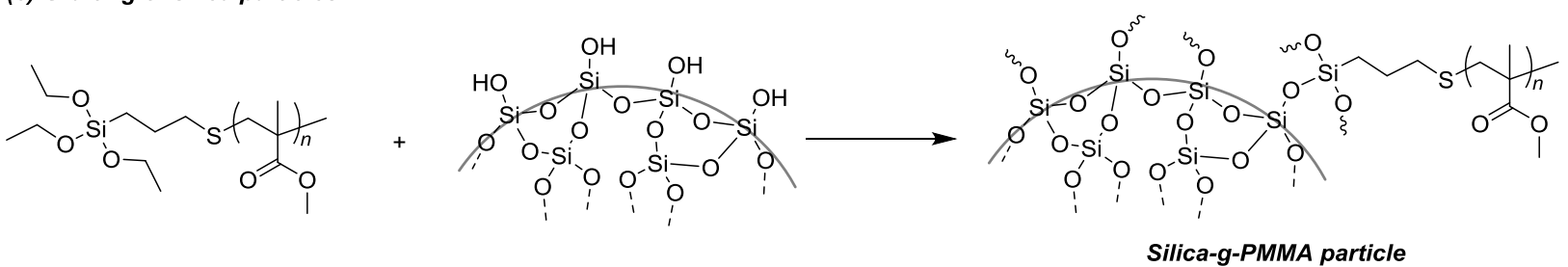

Figure 1: Schematic representation of (a) synthesis of ETS-PMMA, (b) synthesis of silica particles and (c) grafting of silica particles with ETS-PMMA.

\subsection{Preparation of $P M M A /$ silica composites}

The elaborated composites are PMMA with $1 \mathrm{wt} \%$ (=0.5vol \%) of embedded silica. 
Composites were manufactured in several steps (master-batch; blending and injection). The first step of the master-batch consists in producing the desire dispersion:

- Poorly dispersed composites (namely "PMMA-Si-pd" and "PMMA-Si-T-pd") were elaborated using an ultrasonic bath (Retsch UR1) treatment. Silica was ultra-sonicated in acetone for 10 minutes.

Well-dispersed composites (namely "PMMA-Si-wd" and "PMMA-Si-T-wd") were elaborated using a probe sonicator (Branson digital sonifer 250, Danburry, USA). Silica was also sonicated in acetone for 10 minutes. In a previous paper authors confirmed that the sonication time is particles size dependent: smaller particles need higher sonication time to be well dispersed [34]. Moreover, a higher level of rate incorporation necessitate again more sonication time.

Five different formulations were thus realized and are presented in Table 1.

After sonication, Silica was added to the PMMA, previously dissolved in acetone, and mechanically stirred. The blend was casted into a Petri dish under fume hood until the complete solvent evaporation.

Then, the obtained composites were compounded with a twin-screw extruder DSM ${ }^{\circledR}$. The temperature along the screw was controlled at $250^{\circ} \mathrm{C}$ and the extrudate was pelletized. The pellets were dried under vacuum at $80{ }^{\circ} \mathrm{C}$ at least $12 \mathrm{~h}$ and then injected at $250{ }^{\circ} \mathrm{C}$ with a IM15 Zamak Mercator machine (Skawina, Poland). The mold temperature was kept at $70{ }^{\circ} \mathrm{C}$. Molded samples are tensile dogbones specimens corresponding to the ISO 527-2 type-1BA.

\begin{tabular}{ccccc}
\hline Acronym & Process & PMMA & $\begin{array}{c}\text { 1wt\% pristine } \\
\text { silica }\end{array}$ & $\begin{array}{c}\text { 1wt\% treated } \\
\text { silica }\end{array}$ \\
\hline PMMA & & $\mathrm{X}$ & - & - \\
\hline PMMA/Si-wd & Probe & $\mathrm{X}$ & $\mathrm{X}$ & - \\
PMMA/Si-T-wd & & $\mathrm{X}$ & - & $\mathrm{X}$ \\
\hline PMMA/Si-pd & Bath & $\mathrm{X}$ & $\mathrm{X}$ & - \\
PMMA/Si-T-pd & & $\mathrm{X}$ & - & $\mathrm{X}$ \\
\hline
\end{tabular}

Table 1 : Manufactured composites

\subsection{Characterization}

\subsubsection{Dynamic rheology}


Rheological properties of composites were evaluated by oscillatory characterization on a Twindrive rheometer MCR702 (Anton Paar instrument) equipped with parallel plate geometry (plate diameter $25 \mathrm{~mm}$, gap $1 \mathrm{~mm}$ ). All experiments were carried out at $230^{\circ} \mathrm{C}$ under nitrogen to avoid polymer degradation and absorption of moisture. Storage modulus ( $\left.G^{\prime}\right)$, loss modulus ( $\left.G^{\prime}\right)$ and viscosity $(\eta)$ of samples were measured with constant strain oscillatory tests within the linearity limit for all composites: a frequency range $\omega$ of $0.01-100 \mathrm{rad} / \mathrm{s}$ and a strain of $1 \%$ were applied during measurements. A strain sweep was applied to fix the strain limit for the linear viscoelastic response.

Continuous relaxation spectra offer an efficient way to qualify the material viscoelasticity revealing aspects of its behavior that may not be obvious in plots of G' and G". It reflects the molecular movements of macromolecules (correlated to the molecular relaxation time) and can be related to molecular structure and properties. Nevertheless, it is not possible to measure directly a relaxation spectrum. It can be only calculated on the basis of experimental data. G' and G" can be expressed as functions of relaxation spectrum $H$ through the equations (1-2):

$$
\begin{aligned}
G^{\prime}(\omega) & =\int_{-\infty}^{+\infty} H(\tau) \frac{\omega^{2} \tau^{2}}{1+\omega^{2} \tau^{2}} d \ln \tau \\
G^{\prime \prime}(\omega) & =\int_{-\infty}^{+\infty} H(\tau) \frac{\omega \tau}{1+\omega^{2} \tau^{2}} d \ln \tau
\end{aligned}
$$

where $\tau$ is the relaxation time. The evaluation of $H$ from eq. 1-2 is not straightforward since it is solution of a very ill-defined problem. Many publications have studied the best way to obtain it. A nonlinear Tikhonov regularization via a Matlab implementation is used, and the algorithm is fully described in [35]. Times associated with maximum weight in relaxation spectrum plot are related to the microstructure. In particular one can assess that the incorporation of silica into PMMA does not impact matrix molecular mobility. Three tests were performed to ensure reproducibility.

\subsubsection{Dispersion and distribution characterization}

Morphologies of silica composites were observed by Scanning Electron Microscopy (FEI Quanta 200 ESEM) under accelerating voltage of $15 \mathrm{kV}$. Samples were cryo- 
fractured and the cross-section was observed. Dispersion and distribution states of silica particles in PMMA matrix and the impact of their functionalization on microstructure were studied.

\subsubsection{Mechanical tests}

Uniaxial tensile tests were conducted on a Zwick TH010 universal testing machine according to the ISO 527 standard. The crosshead speed is equal to $1 \mathrm{~mm} / \mathrm{min}$ (corresponding to $3 \% \mathrm{~s}^{-1}$ of strain rates). Tensile test is conducted up to the rupture of the specimen. The software used is TestXpert $\circledast$ and allows the recording of time, load and elongation, denoted $\varepsilon$, during the test. The nominal stress is defined by the following expression:

$$
\sigma=\frac{F}{S_{0}}
$$

where $F$ is the recorded load and $S_{0}$ the initial sample section surface.

Three tests were performed to ensure reproducibility.

\subsubsection{Thermogravimetric analysis (TGA)}

TGA was performed with a Perkin Elmer Pyris-1 thermogravimetric analyzer in order to determine the silica weight percent of each composite. Samples $(\sim 10 \mathrm{mg})$ were heated from 30 to $900^{\circ} \mathrm{C}$ with a heating rate of $10^{\circ} \mathrm{C} / \mathrm{min}$ under nitrogen atmosphere.

\subsubsection{Size-exclusion chromatography (SEC)}

It is well known that, for some polymers, particles act on crystallization or nucleation processes. An amorphous matrix has been chosen to avoid any structural modification effect. However, SEC analyses were carried out to ensure PMMA stability. This measurement was performed with a Varian ProStar Model 210 equipped with a RI refractive index detector. Two PLgel $5 \mu \mathrm{m}$ Resipore were used at $70{ }^{\circ} \mathrm{C}$ with a $0.8 \mathrm{~mL} \mathrm{~min}{ }^{-1}$ flow rate of DMF with $0.1 \%$ of $\mathrm{LiBr}$, calibrated using PMMA standards, sample injection amount was typically $20 \mu \mathrm{L}$ at a concentration of 10 $\mathrm{mg} / \mathrm{mL}$. 


\section{Results and discussions}

\subsection{Microstructural characterization and composition}

Qualitative observations of structural and morphological composites evolutions were observed by SEM on cryofractured samples (Figure 2). Micrographs show significant differences between composites manufactured by (a) probe process or (b) bath process. Probe process conducts to a better dispersion/distribution of silica particles. In contrast, large agglomerates $(\sim 20 \mu \mathrm{m})$ were observed for composites manufactured by bath process. The used process highly influence the morphology of the composite [36], [37] and thus its final mechanical properties. Subsequently, composites manufactured by probe process are considered as well-dispersed composites and composites manufactured by bath process are considered as poorlydispersed composites.
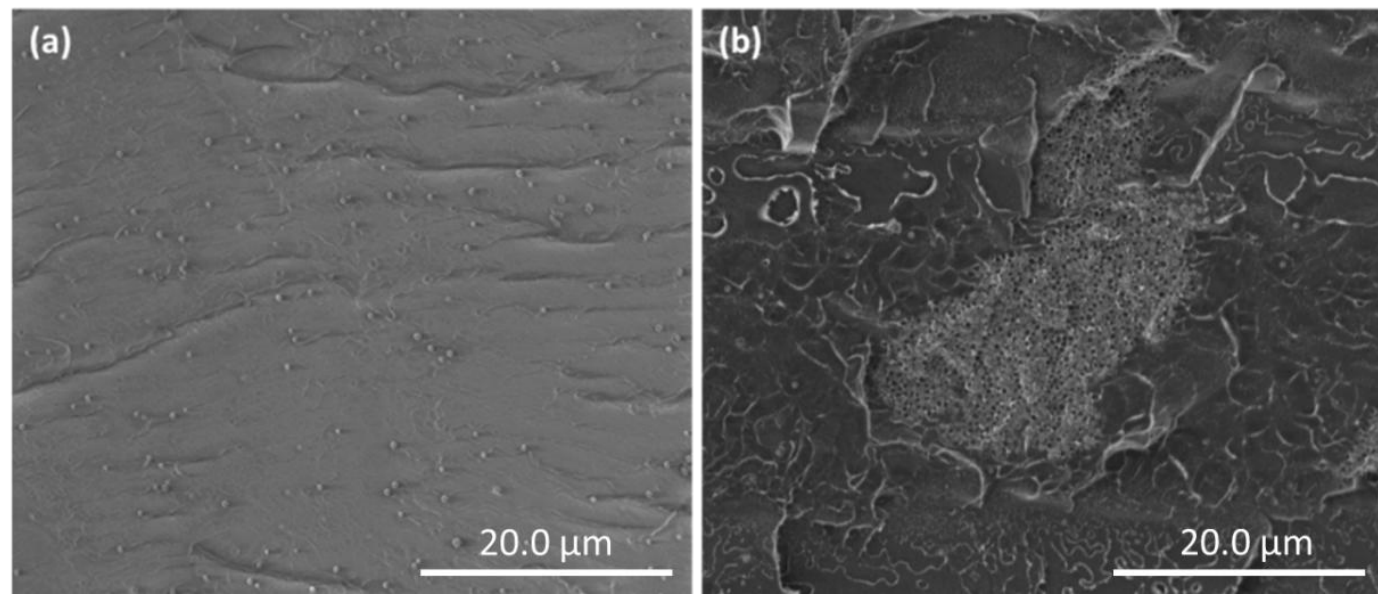

Figure 2: SEM micrographs of PMMA/Silica composites revealing particles dispersion as a function of process (a) probe process (b) bath process

Effective weight percent of embedded silica has to be estimated and considered. Indeed, it is proven that wt\% highly influences the final properties of composites [38][40]. In this study, silica weight fraction of each sample was checked through TGA measurements, after each mechanical test.

Figure 3 represents, as an example, the determination of the silica wt\% of a PMMA/silica-T-pd sample. First results show (see Table 2 which gathers the wt\% of all the samples) a great variability of this weight fraction for all processes. This variability is due to the master-batch, unfortunately this step is essential to safely 
manipulating particles for incorporation in the micro compounder. Samples presented are the extreme cases of $w t \%$.

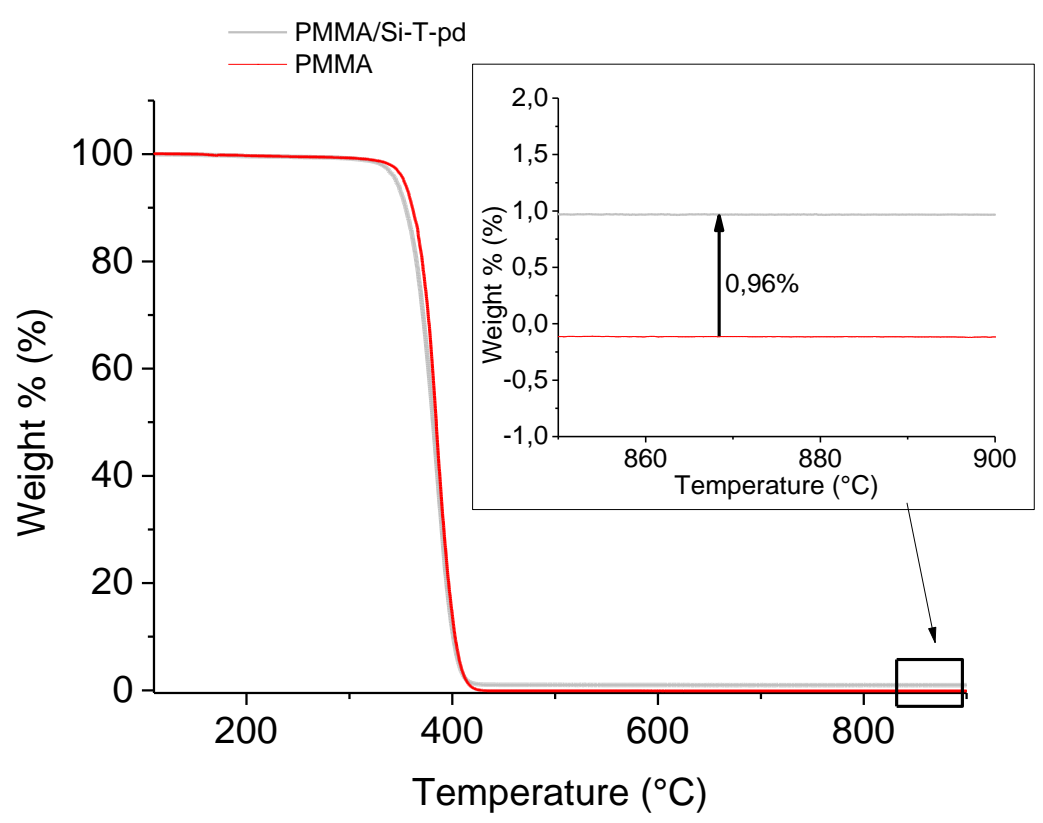

Figure 3: TGA curves of PMMA/silica-T-pd composites (Sample 2), determination of the silica weight percent

\begin{tabular}{lccc}
\hline & Sample 1 & Sample 2 & Sample 3 \\
PMMA/Si-wd & 0.73 & 0.91 & 0.94 \\
PMMA/Si-pd & 0.66 & 0.79 & 1.03 \\
PMMA/Si-T-wd & 0.72 & 0.80 & 1.04 \\
PMMA/Si-T-pd & 0.68 & 0.96 & 1.01 \\
\hline
\end{tabular}

Table 2: Silica wt\% in PMMA composites calculated from TGA results

\subsection{Rheological properties}

The linear viscoelastic properties of molten composites were measured as a function of frequencies from $100 \mathrm{rad} / \mathrm{s}$ to $0.01 \mathrm{rad} / \mathrm{s}$; measurements at very low frequencies are of particular interest for the modelling since the interfacial effects are expected to be predominant. Nevertheless, these measurements are associated to very long experiments questioning thus the in situ polymer thermo-oxidative degradation during experiment [41]. Polymer stability has been checked at $1 \mathrm{~Hz}$ at a prescribed strain 
(3\%) during 60 min to ensure the non-degradation of PMMA. A strain scan was performed until $100 \%$ strain to assess the linear behavior of composite until $3 \%$ of applied strain.

As an example, Figure 4 presents the evolution of dynamic storage modulus versus frequencies for the PMMA/Si-wd composites (3 samples to ensure reproducibility) at $1 \%$ strain. Rheological response is perfectly shifted with the amount of silica. The lower response corresponds to the lower silica weight percent.

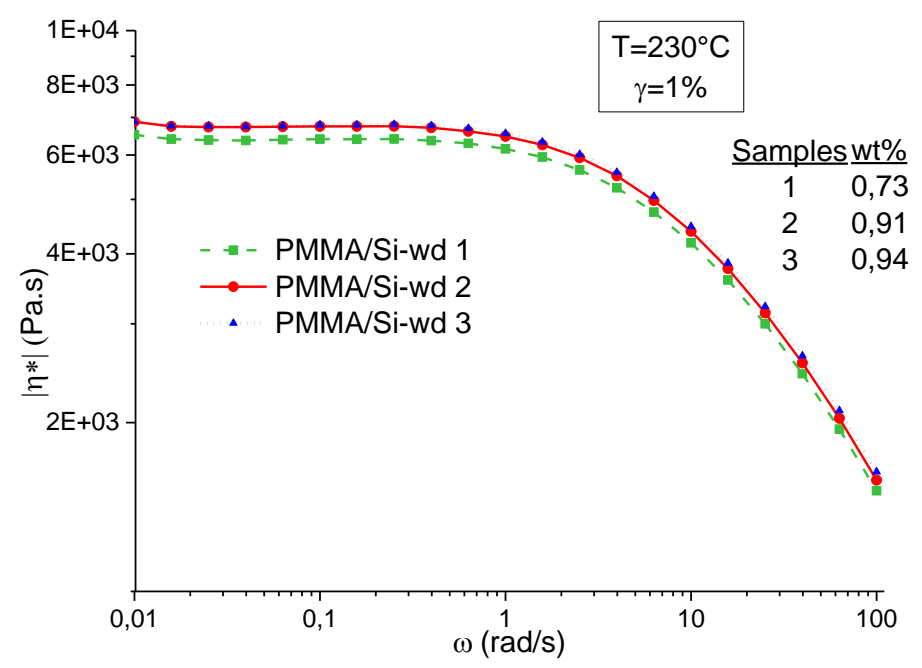

Figure 4: Linear viscoelasticity: variation of the dynamic storage versus frequencies: reproducibility for the PMMA/Si-wd composites

The weighted relaxation spectra $\tau H(\tau)$ versus relaxation time $\tau$ of the neat PMMA and $\mathrm{PMMA} / \mathrm{Si}$ composites at $230^{\circ} \mathrm{C}$ (Figure 5) was obtained from Eqs. (1) and (2). As expected, PMMA depicts a single peak at $0.12 \mathrm{~s}$, which is its characteristic relaxation time. For PMMA/Si-wd composites, two relaxation peaks are observed at about $0.2 \mathrm{~s}$ and after $100 \mathrm{~s}$. The first peak corresponds to the relaxation of PMMA matrix while the second one (which is a slower process) corresponds to the shape relaxation of dispersed silica. This relaxation time could be reached from rheological experiments in the molten stage with imposed frequencies lower than $10^{-2} \mathrm{rad} / \mathrm{s}$. 


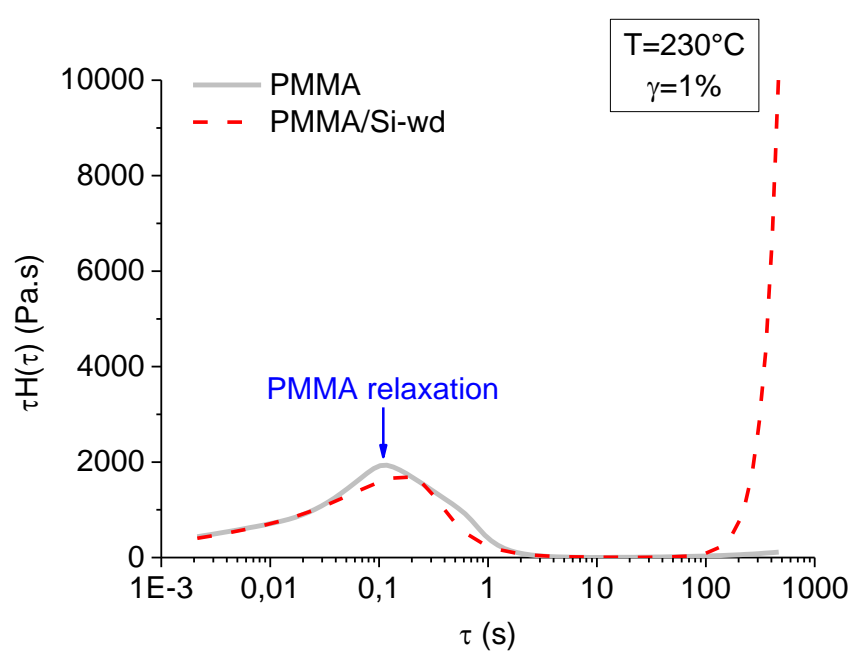

Figure 5: Weighted relaxation spectra for the neat PMMA and PMMA/Si-wd composites at $230^{\circ} \mathrm{C}$.

The first relaxation time is slightly shifted to higher values with the incorporation of silica independently of its dispersion and functionalization. This means that the PMMA matrix is not chemically modified with the incorporation of silica: in average, its chains mobility remains unchanged. SEC analyses were carried out to confirm this conclusion. Results show there is no structural change of PMMA after silica incorporation (average $M_{n}=50000 \mathrm{~g} / \mathrm{mol}$ ).

\subsection{Mechanical testing}

Three samples of each material have been tested through uniaxial tensile tests. Results are presented in Figure 6. It seems that, in average, the global mechanical behavior (except the breakage) is the same for all the tested composites. The major difference is the elongation at break which is lower for the PMMA/Si-pd composites and higher for the PMMA/Si-T-wd composites. The addition of silica into PMMA leads to increase the strength and to lower the elongation at break (see Table 3), excepted for composites with embedded well-dispersed and functionalized silica. The value of standard deviations reflects the variability in the breaking response, attributed to material inhomogeneity. 


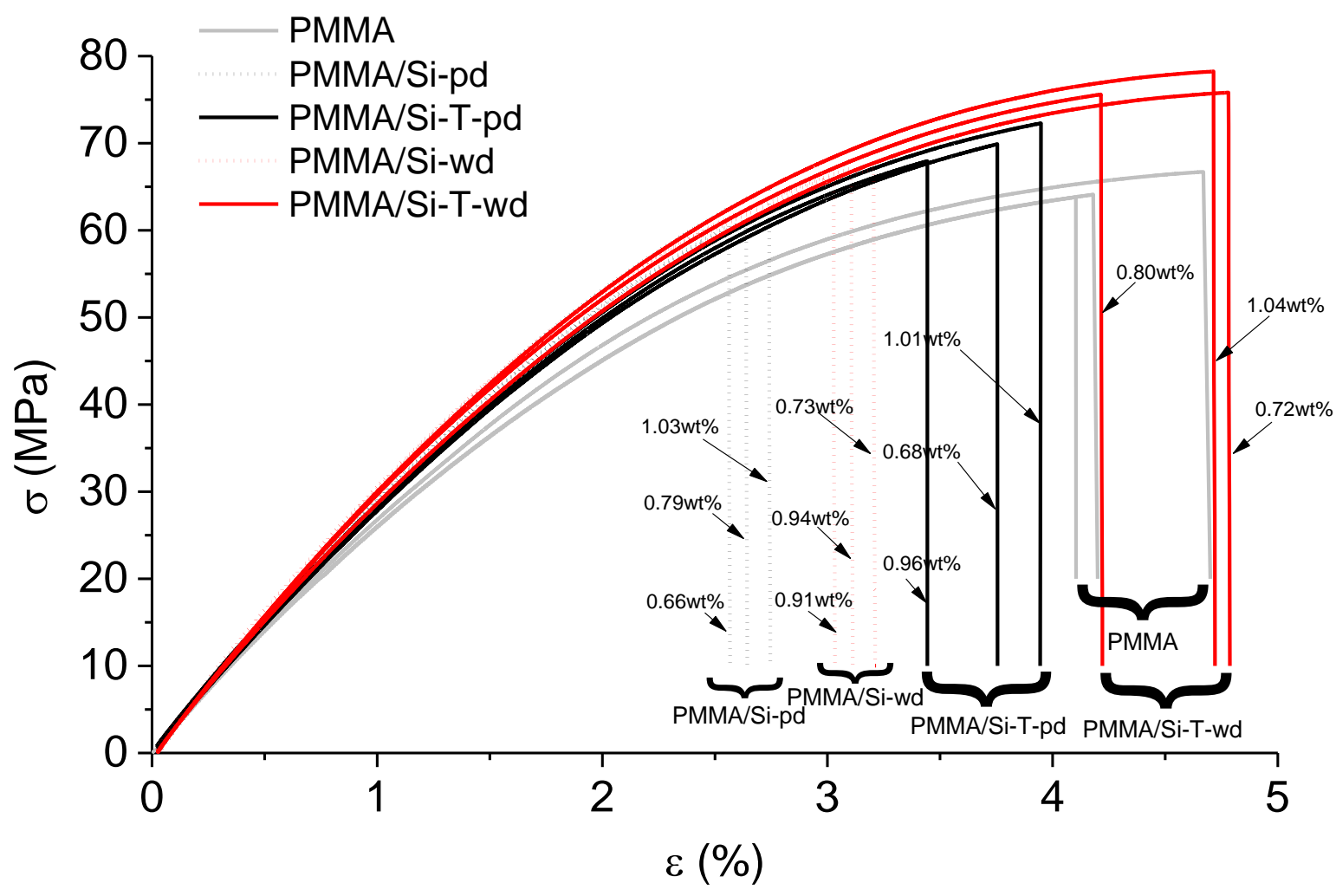

Figure 6: Uniaxial tensile responses for PMMA and PMMA/Si composites

Stresses at break of PMMA and composites are presented in Table 3. These results reveal that finally if the sought properties are the improvement of the breakage, then the questioning on the functionalization interest arises. Indeed, according to the results and in view of the functionalization cost, it is ultimately more advantageous to manufactured composites with well-dispersed and unmodified silica particles instead of composites with poorly-dispersed and modified silica particles. On the other hand, it is clearly proved that composite with modified and well-dispersed silica embedded is the better case.

\begin{tabular}{lcccc}
\hline & $\begin{array}{c}\text { Elongation at } \\
\text { break (\%) }\end{array}$ & $\begin{array}{c}\text { Standard } \\
\text { deviation }\end{array}$ & $\begin{array}{c}\text { Stress at } \\
\text { break } \\
(\mathrm{MPa})\end{array}$ & $\begin{array}{c}\text { Standard } \\
\text { deviation }\end{array}$ \\
\hline PMMA & 4.33 & 0.32 & 65 & 1.6 \\
PMMA/Si-wd & 3.11 & 0.08 & 67 & 2.4
\end{tabular}




$\begin{array}{lllll}\text { PMMA/Si-pd } & 2.65 & 0.09 & 62 & 0.0 \\ \begin{array}{l}\text { PMMA/Si-T- } \\ \text { wd }\end{array} & 4.57 & 0.32 & 77 & 2.2 \\ \begin{array}{l}\text { PMMA/Si-T- } \\ \text { pd }\end{array} & 3.67 & 0.25 & 70 & 1.4\end{array}$

Table 3: Elongation at break and stress at break for PMMA and PMMA/Si composites

\section{Modelling and discussion}

\subsection{Matrix Behavior}

As a first approximation, the global PMMA behavior is expected to follow an elastoviscoplastic evolution. The stress and strain represented in Figure 6 are part of tensorial stress and strain representation $\boldsymbol{\varepsilon}, \boldsymbol{\sigma}$. The plastic flow potential $f$ is written as:

$$
f=\sigma_{\text {mises }}-R_{0}
$$

where $\sigma_{\text {mises }}$ is the Von Mises stress and $R_{0}$ is a material parameter.

The viscoplastic strain tensor is written with a Norton law:

$$
\dot{\boldsymbol{\varepsilon}}=(1-p) \dot{p} \boldsymbol{n}_{*}, \quad \boldsymbol{n}_{*}=\frac{\partial \sigma_{\text {mises }}}{\partial \boldsymbol{\sigma}}, \quad \dot{p}=\left(\frac{f}{K}\right)^{n}
$$

where $p$ is the effective plastic strain, $n$ and $K$ are material parameters. Bold letters refer to tensorial values.

The PMMA stress evaluation is shown in terms of scripts written in Matlab®. Equations (4-5) are implemented via an incremental formulation leading to the value of the uniaxial stress from strain tensor components values. Strains from uniaxial tensile tests are used as input in the modelling offering the possibility to compare experimental (PMMA in Figure 6) and numerical data.

A minimization algorithm based on Levenberg Marquard algorithm lead to a set of parameters (given in Table 4) that conduct to a good accordance between analytical and experimental stress/strain curve (Figure 7). The number of iterations and residue value are reported in Figure 7. 


\begin{tabular}{cc|cc}
\hline \multicolumn{2}{c|}{ Silica } & \multicolumn{2}{c}{ PMMA } \\
\hline $\mathrm{E}(\mathrm{GPa})$ & 70 & $\mathrm{E}(\mathrm{GPa})$ & 3.2 \\
$v$ & 0.3 & $v$ & 0.42 \\
& & $n$, K (GPa.s) & $0.7,4.7$ \\
& & $R_{0}(\mathrm{MPa})$ & 17.3 \\
\multicolumn{2}{c}{ Table 4: Material parameters for PMMA and Silica }
\end{tabular}

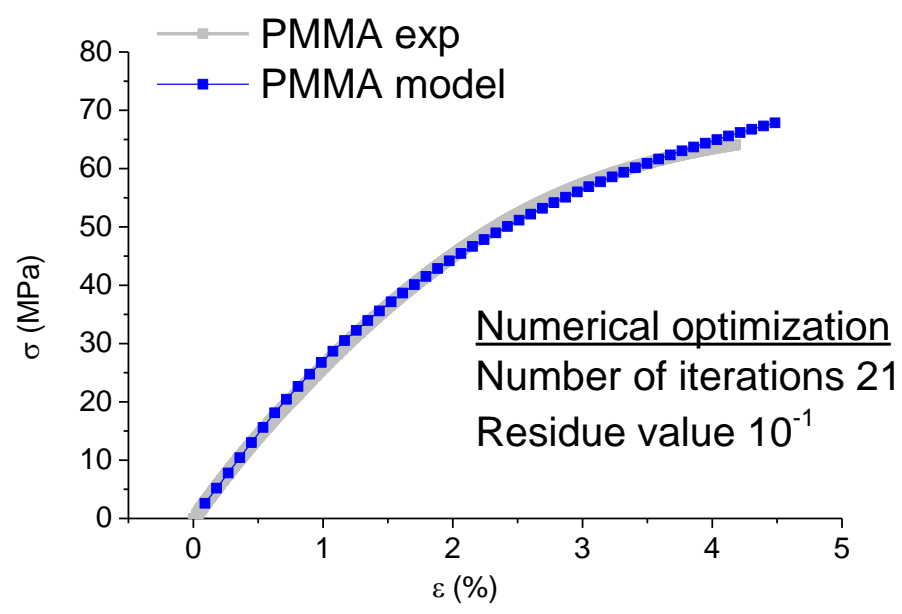

Figure 7: Modelling of PMMA, stress vs. strain curve

\subsection{Predictive modelling of the composites}

Numerical simulations were carried out using FE software Zebulon, developed at Mines Paristech [42]. Material parameters associated to PMMA are listed in Table 4. The silica particles are assumed to be isotropic elastic, with a global behavior fully describe through elastic data such as Young Modulus and Poisson's ratio (Table 4, values extracted from literature [43]).

\subsubsection{Perfect interface matrix/filler}

The interface between matrix and fillers is firstly assumed to be perfect (assuming a continuous displacement through the interface) and properties of phases are described through the set of parameters given in Table 4. As the PMMA properties 
are not such impacted by the fillers (see section 3.2), it was assumed that the properties of PMMA matrix into the composite are similar to those of neat PMMA.

An example of 3D structure (generated via DIGIMAT software), in which silica fillers are spherical, monodispersed and well dispersed into the matrix is given in Figure 8. Mesh microstructure and boundary conditions are given in this figure. The size of the representative elementary volume (REV) and the number of elements correspond to minimal values leading to a convergent stress/strain numerical data.
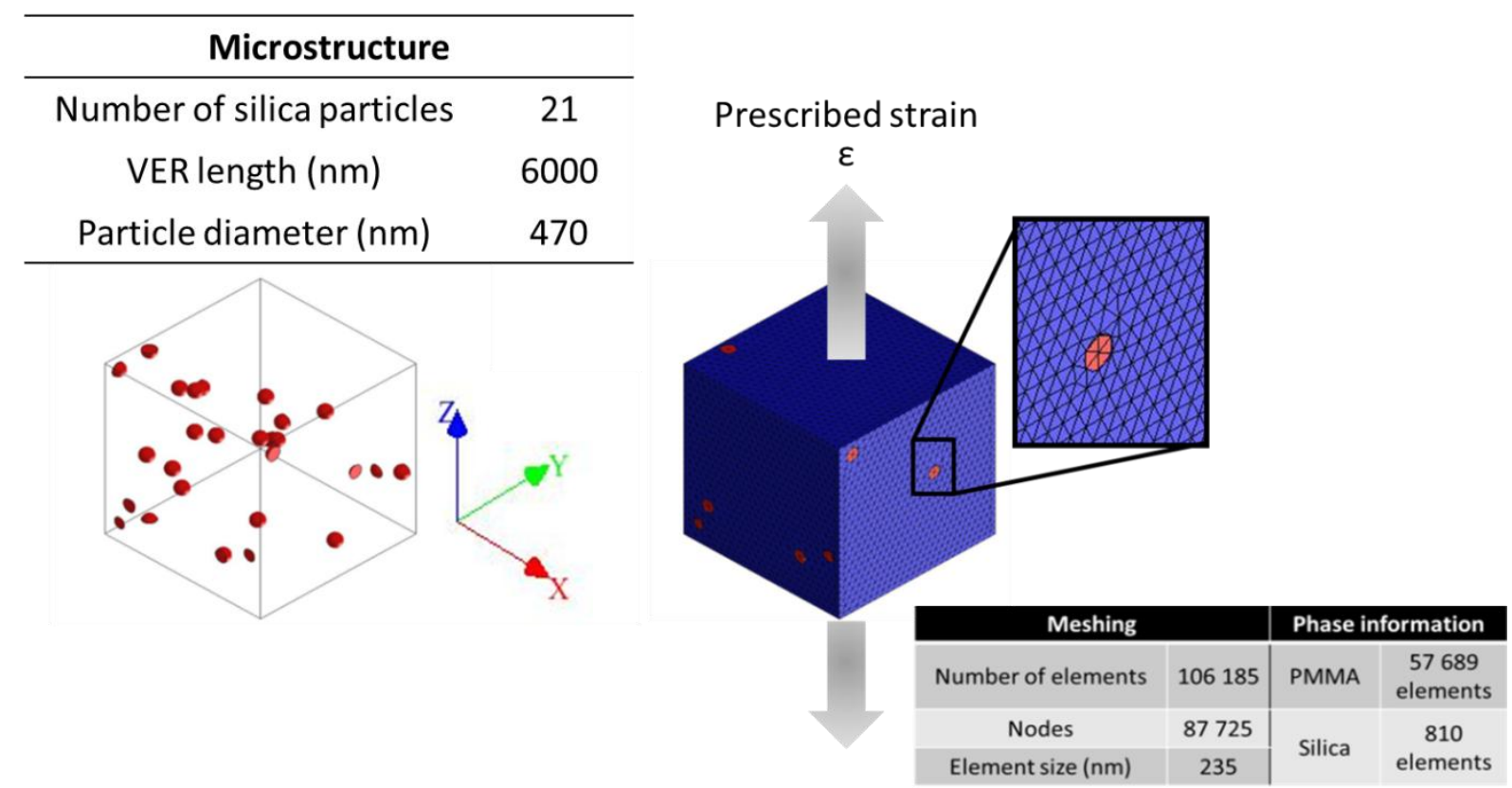

Figure 8: Digimat microstructure and boundary conditions

Comparison between numerical and experiments is given in Figure 9 for PMMA/Si composite. As a first result, input materials parameters lead to a prediction similar to the one of neat PMMA. This means that the amount of silica (1\%wt) is numerically insufficient to enhance PMMA mechanical properties. However, an enhancement of PMMA properties is experimentally observed, as shows the PMMA/Si-T-wd curve. It is already well known that classical micromechanical models are not able to describe mechanical response of nanocomposites. These models do not consider the interactions between filler and matrix at their interface. For nanocomposites, the mechanical property enhancement not only depends on constituent properties, constituent volume fraction, inclusion shapes or orientation and state of dispersion, but also on the interaction between the filler and the matrix. The existence of an interphase between the nanofillers and the matrix is widely accepted [9], [44]. This interphase is a transition region of nanometric to micrometric size, where the 
mechanical and physical properties change from the properties of filler to the properties of the matrix. To extend their applicability to nanocomposites, analytical models were improved to consider coated inclusions [10], [26]-[28], [45]. Even if in this work, silica diameter size is upper than classical nanoparticles, this nanosize effect is experimentally observed.

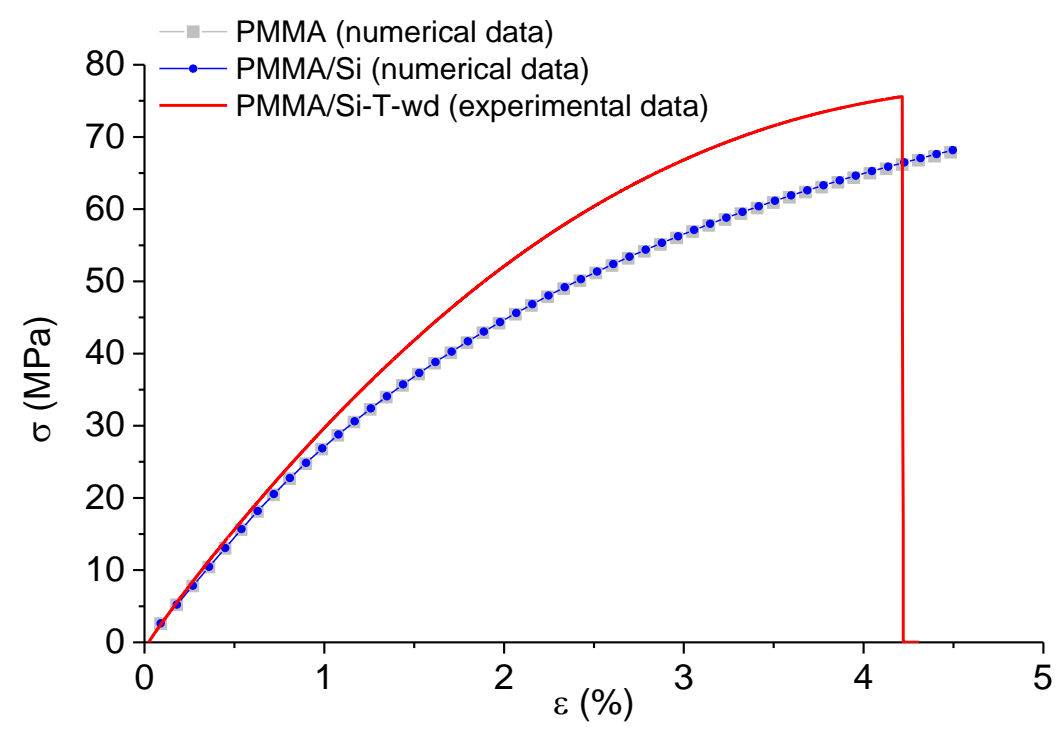

Figure 9: Finite element modelling of PMMA/Si composite

\subsubsection{Existence of an interphase matrix/filler}

\subsubsection{Single filler modelling}

The modelling can be an acceptable technique to consider the interphase in polymer composites without any specific and practical method for evaluation of interfacial properties. So far, nanocomposite structure has been simply regarded as a three phase multi-inclusions based on the continuum model. The modified polymer region surrounding the particles is considered as an independent material that differs from PMMA and silica materials. The choice in this article is to fix the interphase thickness $e_{\text {inter }}$ and to fit its elastic properties. In this interphase, the polymer molecular mobility is assumed to be modified by the particle independently of its functionalization. The interphase thickness is arbitrary fixed to particle size diameter as in [13], [14], [19], [46], corresponding to $13 \%$ in volume of the REV. Two cases are considered (Figure 10): 
- perfect continuous interface between silica/interphase and matrix/interphase (functionalized silica case).

- decohesive interface between silica and interphase but continuous interface between matrix and interphase : in this case a Cristfield debonding model, suitable to describe interface debonding, is used [47]. This model is described through a scalar variable $\lambda$ which characterizes the relative crack opening between matrix and fillers:

$$
\lambda=\frac{1}{\eta} \frac{\langle\kappa\rangle}{1+\langle\kappa\rangle}, \kappa=\sqrt{\left(\frac{\left\langle u_{n}\right\rangle}{u_{0 n}}\right)^{2}+\left(\frac{\left\langle u_{t}\right\rangle}{u_{0 t}}\right)^{2}}-1, \eta=1-\frac{u_{0 n}}{\delta_{n}}=1-\frac{u_{0 t}}{\delta_{t}}
$$

where $\langle$.$\rangle holds for the positive value of the quantity. With respect to the interface$ normal $\boldsymbol{n}, u_{n}=\boldsymbol{u} . \boldsymbol{n}$ and $\boldsymbol{u}_{t}=\boldsymbol{u}-u_{n} \boldsymbol{n}$ denote, respectively, the normal and shear opening displacements and $\delta_{n}, \delta_{t}$ the corresponding maximum allowable values of their norms. The parameters $u_{0 n}, u_{0 t}$ denote repectively the opening displacements corresponding to the maximum cohesive traction of the normal and shear components. $\lambda$ increases from 0 (no damage) to 1 (for a broken element). $\lambda_{\max }$ is the maximum value reached by $\lambda$. The normal and shear components of the cohesive traction $\boldsymbol{T}=T_{n} \boldsymbol{n}+\boldsymbol{T}_{t}$ are defined by:

$$
T_{n}=\frac{u_{n}}{u_{0 n}} F\left(\lambda_{\max }\right), \boldsymbol{T}_{t}=\alpha \frac{\boldsymbol{u}_{t}}{u_{0 t}} F\left(\lambda_{\max }\right), F(\lambda)=\sigma_{\max }(1-\lambda)
$$

with $\alpha$ a constant representing the relative magnitude of $\left\|\boldsymbol{T}_{t}\right\|$ with respect to $T_{n}$, and $\sigma_{\text {max }}$ the maximum stress allowable by the element. For the compressive case

$$
T_{n}=\alpha_{c} \frac{u_{n}}{u_{0 n}} F(0)
$$

with $\alpha_{c}$ a penalization factor.

$\left(\sigma_{\max }, u_{0 n}, u_{0 t}, \delta_{n}, \delta_{t}, \alpha, \alpha_{c}\right)$ parameters are chosen arbitrarily to model a weak interface $\left(\sigma_{\max }=0\right.$, Figure 10). 


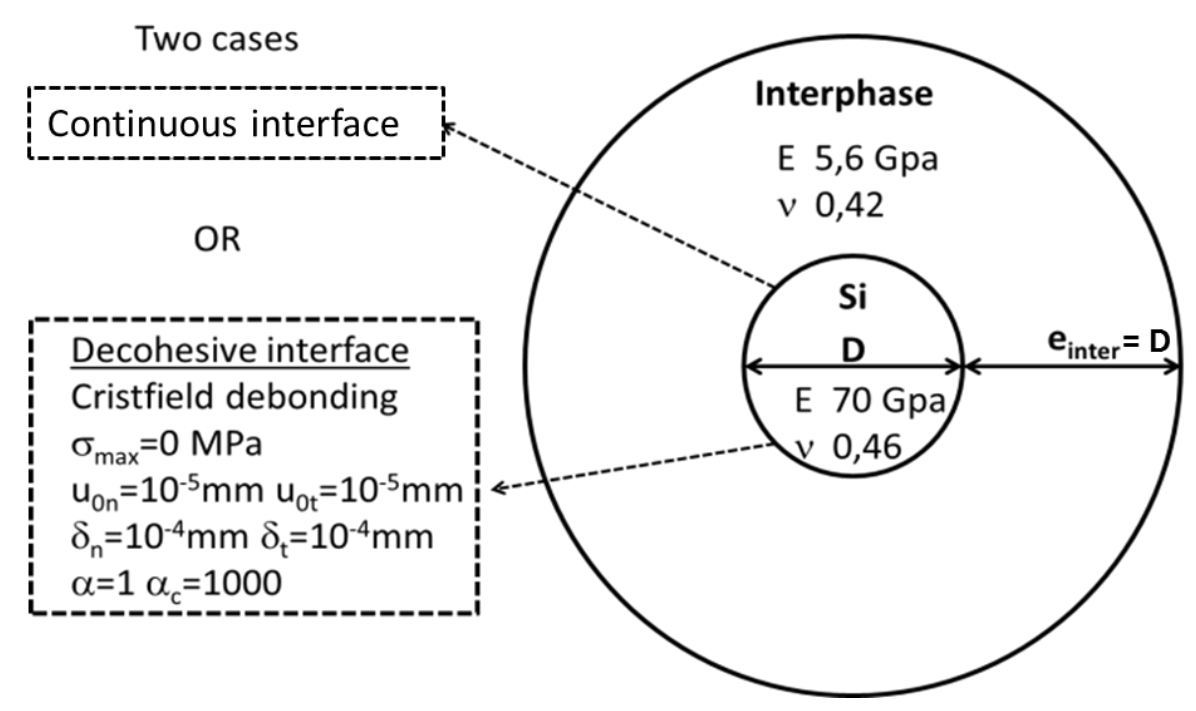

Figure 10: Interphase modelling for PMMA/Si model composite

SEM micrographs in Figure 11 confirm the previous consideration. The weaknesses areas for the PMMA with pristine silica (Figure 11 (a)) are in the vicinity of the particle in the interphase (decohesive interface). Black holes, refer to eroded particles, are much more present for pristine silica. In the case of PMMA with functionalized silica (Figure 11 (b)), interface between interphase and silica seems to be continuous. 

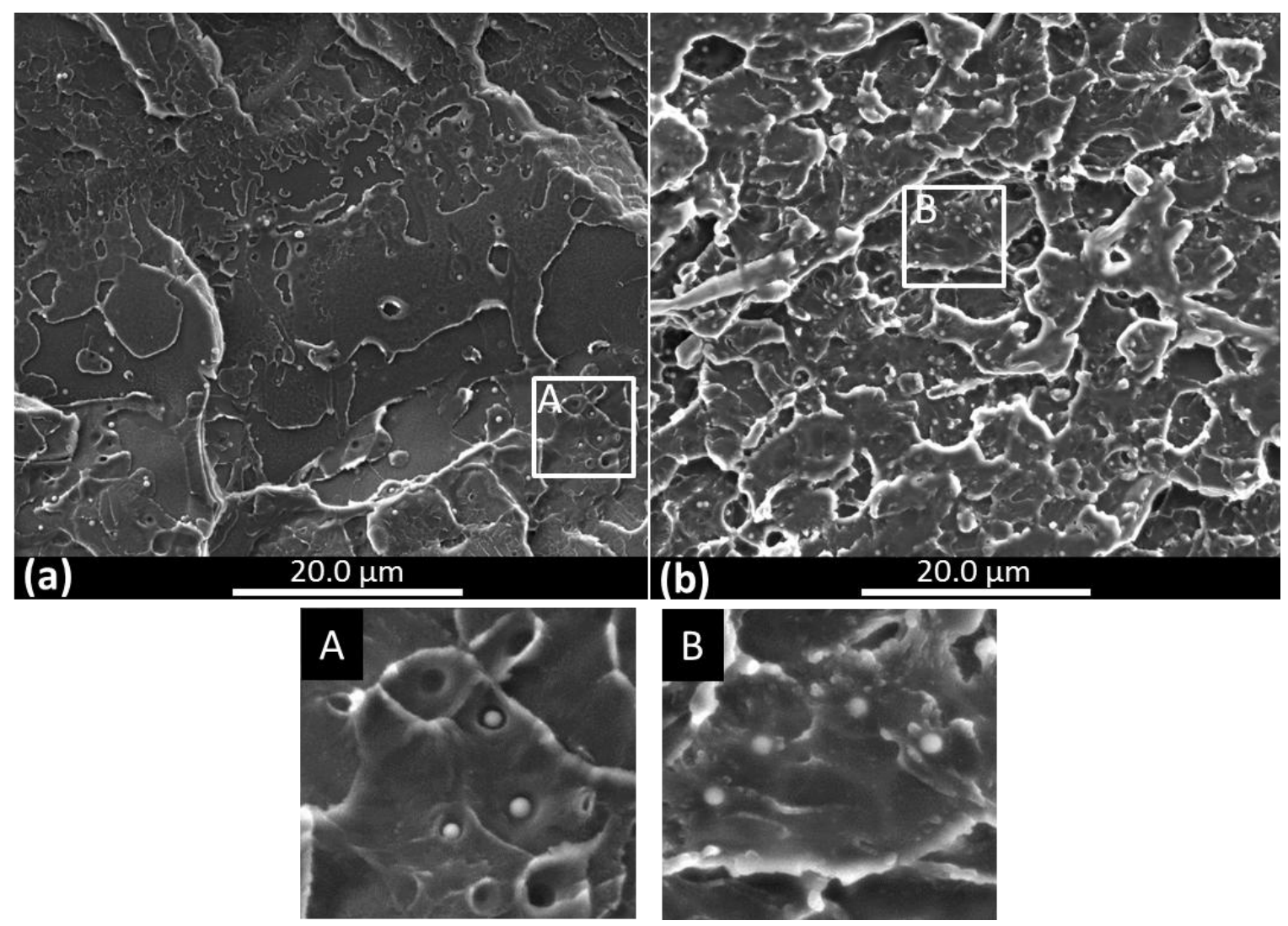

Figure 11: Micrographs of a) PMMA/pristine silica and b) PMMA/functionalized silica composites

In a first modelling, a single particle in a PMMA REV is considered: an axisymmetric assumption is supposed and only $1 / 4$ of structure is represented (see Figure 12). The cylinder length and radius are chosen to maintain the effective weight percent to $1 \%$. Regarding the low weight fraction of silica, if silica is well dispersed into the matrix, it seems reasonable to consider that fillers do not interact with each other. This assumption will be validated in the latest section. In this simulation displacement continuity is assumed at the interface silica/interphase/PMMA. The REV is subjected to a top displacement value of $\varepsilon L$, where $L$ is the square length of the REV.

The numerical stress response which corresponds to the average Von Mises Stresses of the REV elements will be compared to experimental stress recorded during the tensile test performed on PMMA/Si-T-wd composites. For this composite, the adhesion silica/PMMA is assumed to be improved by the functionalization in comparison with pristine silica justifying the comparison with a modeling based on perfect continuous interface between silica/interphase and matrix/interphase. The 
elastic interphase properties are fixed from the better correspondence between the experimental and numerical stresses.

The optimization procedure quickly converges towards to a Young modulus of 5.6 GPa and a Poisson ratio of 0.42 for the interphase area. These latest elastic values could be reached if the molecular weight $\left(\mathrm{M}_{\mathrm{w}}\right)$ of the matrix is modified in the vicinity of the fillers. The effect of the polymer molecular weight has been already observed by some authors (for example an increase of about 2 for the young modulus as $M_{w}$ is divided by $10[48])$.

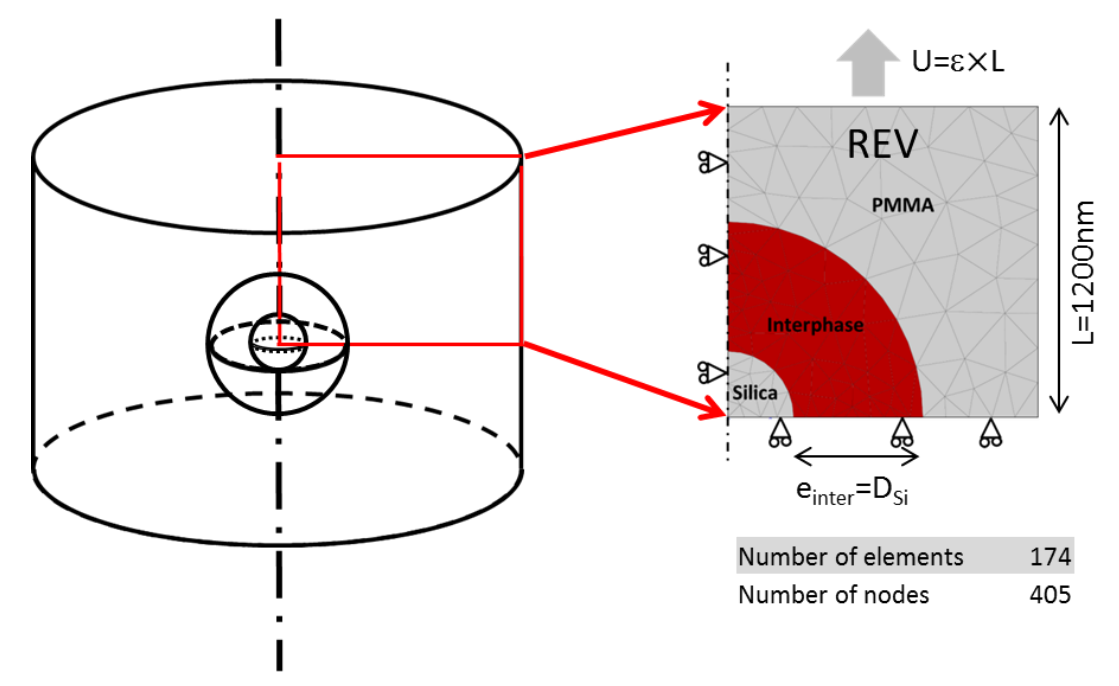

Figure 12: Representative elementary volume and boundary conditions for the finite elements computation.

The corresponding macroscopic response is presented in Figure 13, namely "one element simulation (perfect interface)". A thinner interphase would have conduct to higher value for Young modulus [49].

The model well reproduced the experimental stress/strain response until 3\% of strain. After that the modelling overestimates experiments (Figure 13). A damageable behavior of the interphase area would be more convenient to obtain a better fit.

From this REV, a second simulation is performed for comparison with a decohesive interface between silica and interphase. This simulation will be helpful to understand the impact of functionalization of fillers. The stress/strain response is given in Figure 13 called "one element simulation (decohesive interface)". The level of macroscopic stress is almost unaffected by the quality of silica/PMMA interface. This observation is in accordance with experiments for which ultimate properties are mainly impacted by the functionalization. 


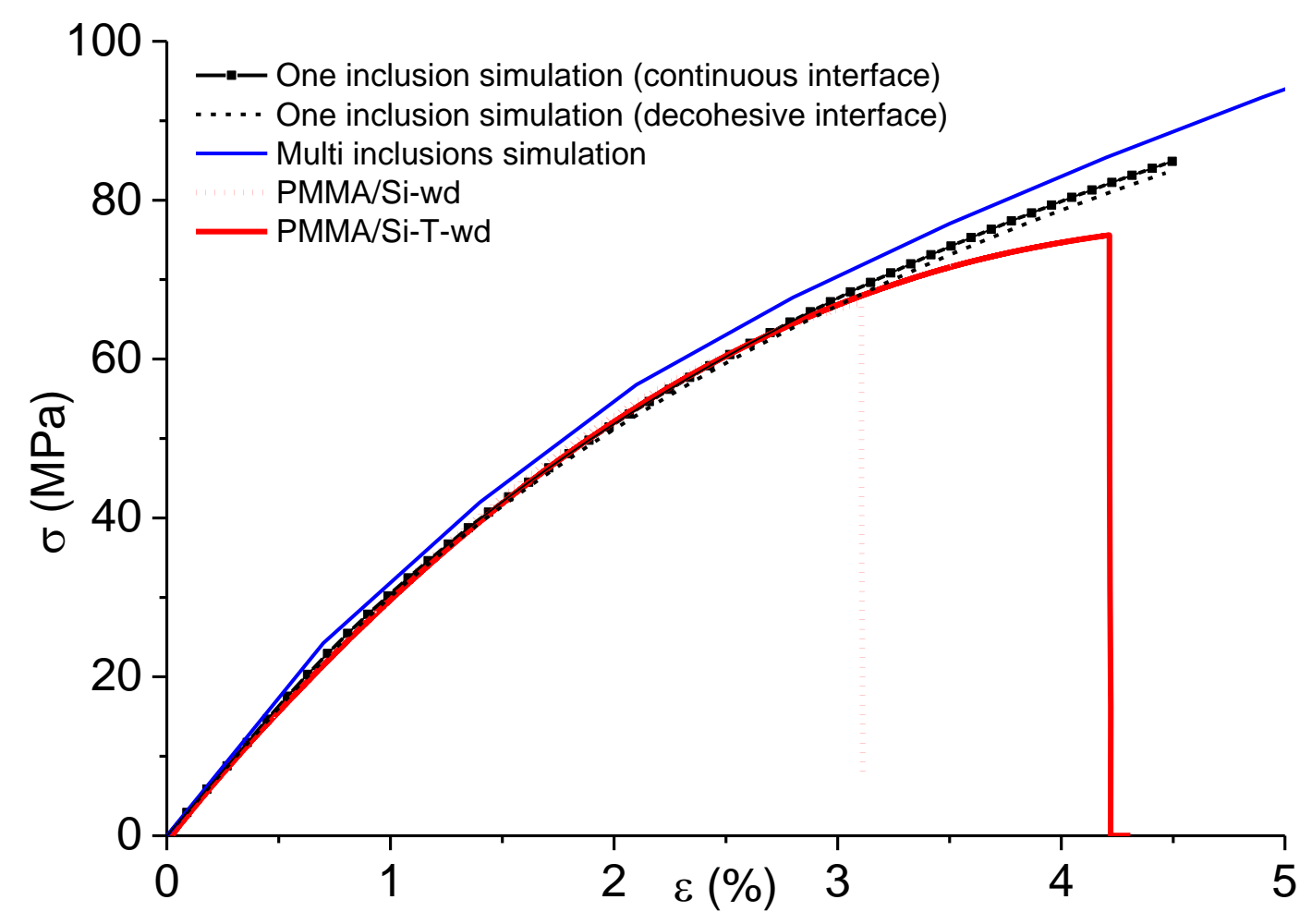

Figure 13: Uniaxial tensile responses for the PMMA/Si composites: comparison between experiments and simulations

The previous modelling was performed on a perfect composite for which the silica weight ratio was fixed to $1 \mathrm{wt} \%$. The reality is that weight fraction is variable (see section 3.1). The numerical simulation enables to evaluate the impact of this weight variation. The simulations results are presented in Figure 14 for various weight ratios (from 0.64 to $1.09 w \%$ ). Numerical responses are strongly affected by the weight fractions and the experimental results are framed by these responses. 


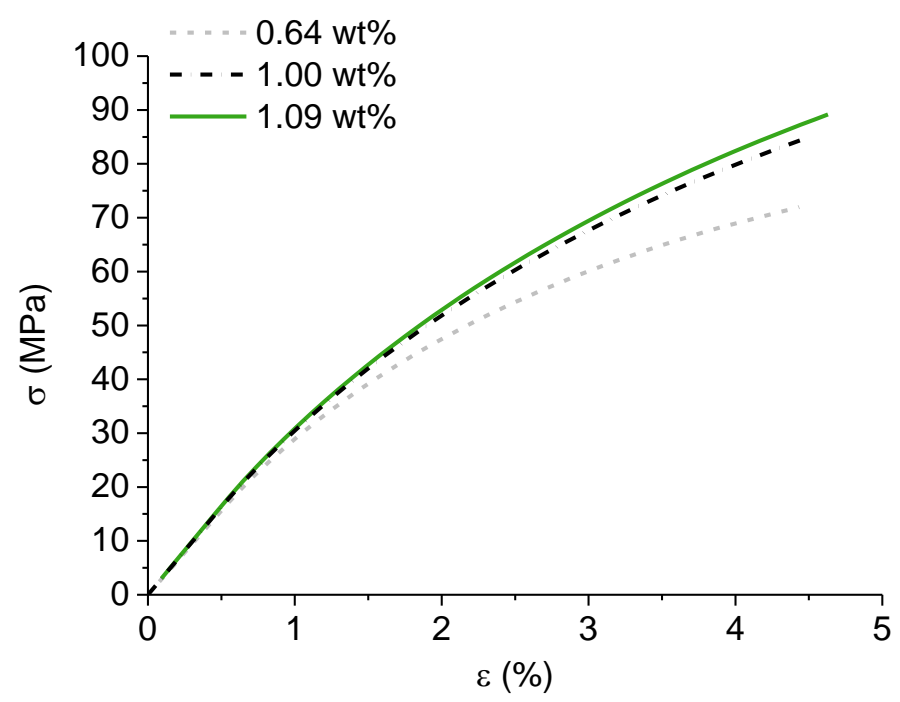

Figure 14: Numerical uniaxial tensile responses for the PMMA/Si composites (one filler): comparison between several fills rates

As an example, Von Mises stress cartography is given in

Figure 15 for both configurations (continuous and decohesive) at a step of the simulation corresponding to $3.1 \%$ of applied strain. This latest value is chosen from experimental data of Table 3 as; it corresponds to the minimal ultimate strain between PMMA/Si-T-wd and PMMA/Si-wd. The stress field in the interphase is very different depending on the simulations, as it corresponds to a multi axial stress field. The Von Mises stress is mainly concentrated in the silica and above the silica in the continuous case while stress concentration are more located in the interphase and matrix areas in the decohesive case. According to the observe cumulative frequency associated to these stress cartographies in both cases (Figure 16(a)), the cumulative number of elements that have reached or exceeding critical Von Mises stresses values (up to $\sigma_{u}=65 \mathrm{MPa}$ ) is higher in the PMMA area (54\%) in comparison with the interphase area (20\%). Moreover in the PMMA area the decohesive simulation leads to increase the number of elements with a high level of Von Mises stress even if this difference appears far from $\sigma_{u}$ thus questioning the modeling validity within this stress range. This observation could explain the lower value of ultimate strain for the non-functionalized composite, even if the level of stress inside PMMA matrix exceeds the experimental ultimate stress and strain that PMMA can undergo. This conclusion is confirmed by the cumulative plastic strain plotted as a histogram in Figure 16(b). Even if the average value of effective plastic strain is almost the same, in the 
decohesive simulation the value reached is much higher in comparison with the continuous interface thereby justifying a premature breakage of the nonfunctionalized composites.

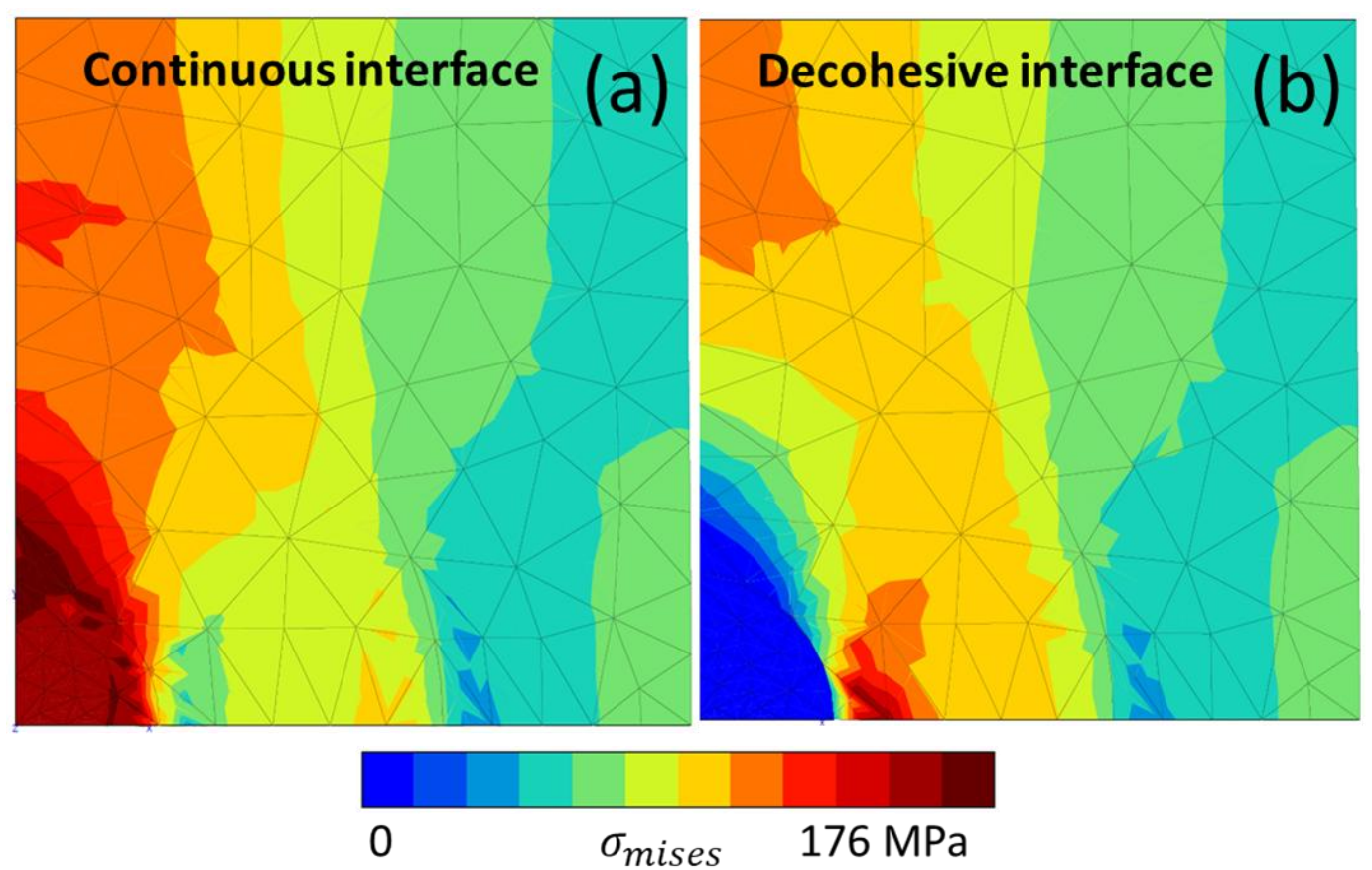

Figure 15: Von Mises stress cartography for a continuous interface (a) and a decohesive interface (b) corresponding to $3.1 \%$ of macroscopic strain 


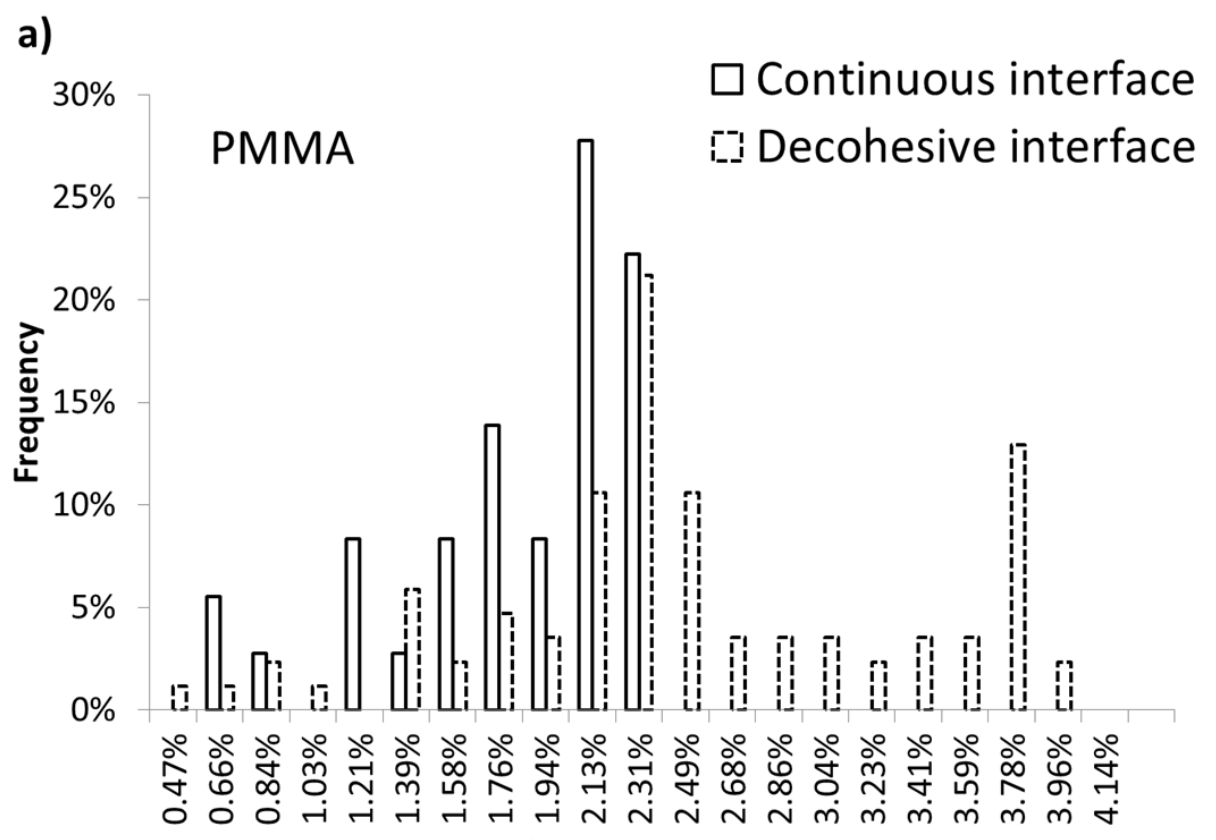

Effective plastic strain

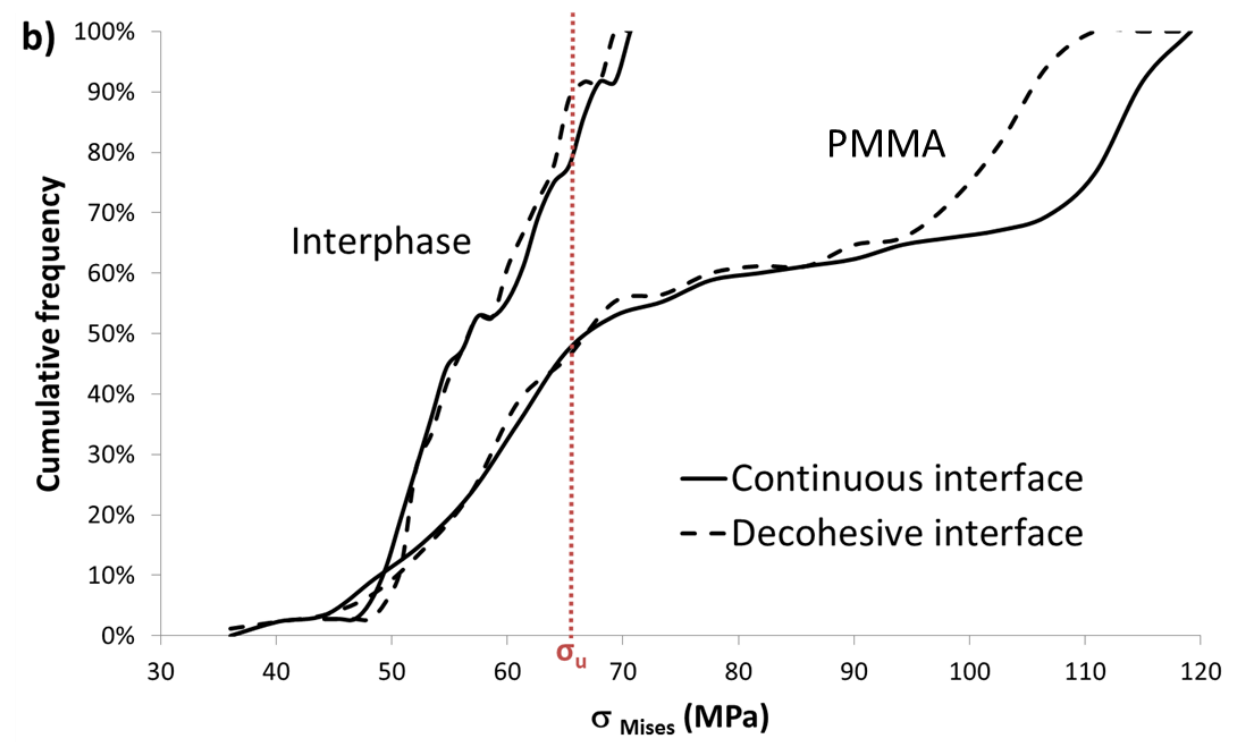

Figure 16: Cumulative frequency of Von Mises stresses of finite elements in the interphase and PMMA area (a) Frequency of effective plastic strain of finite elements in PMMA area (b) corresponding to $3.1 \%$ of macroscopic strain

\subsubsection{Multi fillers modelling to consider dispersion}

The influence of fillers/matrix affinity has been studied in the previous part. The impact of silica dispersion is the key point of this part. Two kinds of microstructures have been generated via DIGIMAT software: the first one is related to a well dispersed particles configuration and the second one is an agglomerated particles 
configuration (Figure 17). Microstructures were generated four times to ensure the reproducibility depending on the particle positions. For each formulation the interface silica/interphase is considered continuous and the interphase properties are those defined in the previous part. These new simulations enable a comparison between PMMA/Si-T-wd and PMMA/Si-T-pd.
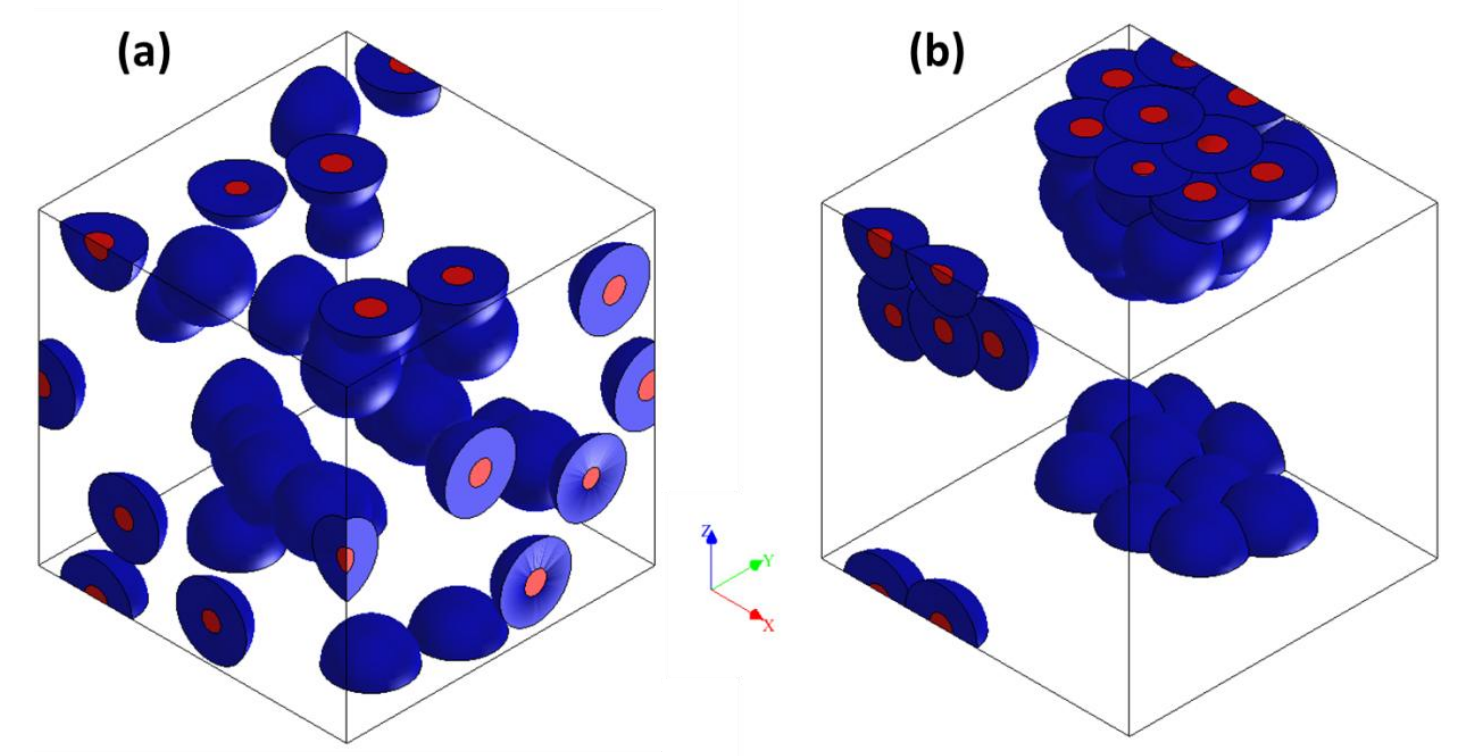

Figure 17: PMMA/Si composites: two model microstructures (a) well-dispersed and (b) poorly-dispersed

Results of modelling are presented in Figure 18. The agglomerated microstructure leads to a slightly higher macroscopic response possibly due to trapped matrix which does not contribute to the global macroscopic composite response [9]. Nevertheless considering the low weight ratio, the influence of dispersion is not significant, beside ultimate properties as already presented by Peng et al [50]. Regarding the ultimate properties (Table 3), the elongation at break is higher for the composite with well dispersed particles, functionalized or not. This is in agreement with the previous local REV observations (Figure 13): even if the macroscopic response is quite the same, the local levels of stress responses in both REV are fundamentally different. For the poorly dispersed composites, the decrease of the interparticular distance lead to enhance the level of triaxiality, assumed potential damage source. 


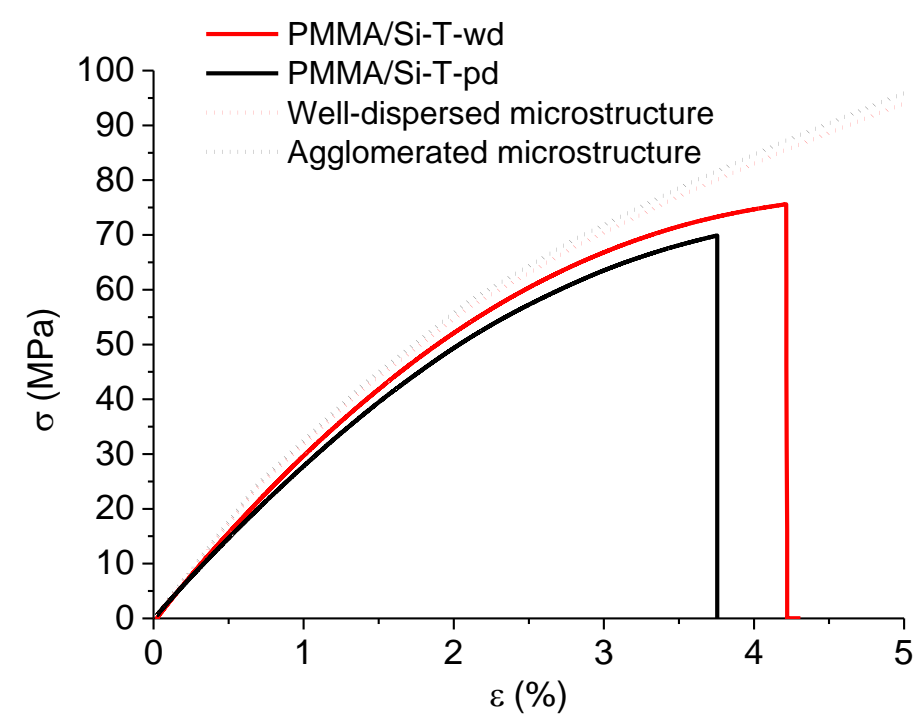

Figure 18: Uniaxial tensile responses for the PMMA/Si composites: influence of silica dispersion

\section{Conclusions}

Mechanical properties of PMMA based composites were studied in this work. Experiments (uniaxial tensile tests) were carried out on PMMA and PMMA filled with $1 \%$ by weight of silica particles. Silica particles surface was for some cases functionalized with PMMA chains. Dispersion state of silica into the matrix is controlled via two different procedures. At first, global measured values (load during the test) lead, for the neat PMMA, to a set of mechanical parameters describing an elastoplastic model that well reproduce the experimental stress/strain curve. Knowing the fillers mechanical properties, PMMA/ silica is considered as a three phases composite (Matrix/fillers/interphase) as the matrix is supposed to be modify in the vicinity of the silica. The use of a cohesive model zone at the interface Interphase/Silica enables the consideration of silica functionalization. A FE modelling is used both to predict composites behavior and to understand the damage micromechanisms involved during a uniaxial tensile test. Several conclusions can be drawn from this study:

1. Experimentally, even if our filler is not strictly speaking a nanofiller, the "nano effect" is observable for a low level of incorporation where both Young 
modulus and yield stress are improved, leading to introduce in the modeling an interphase area stiffer than the matrix,

2. Numerically, the overall properties (excepted the breakage) are not sensitive to fillers functionalization nor to their dispersion,

3. Regarding the ultimate properties, composites follow this classification: poorly dispersed and pristine silica < well dispersed and pristine silica < poorly dispersed and functionalized silica $<$ well dispersed and functionalized silica.

For pristine or modified silica particles a careful dispersion protocol is required to obtain an improvement of mechanical properties. Even if the surface modification of silica, which increases the compatibility with the polymer matrix, gives better mechanical properties, according to this study, a careful dispersion protocol seems to be advantageous when the functionalization cannot be envisaged.

\section{Acknowledgements}

This work was supported by the GDR Polynano and IMT mines Alès. The authors would like to thank Dr. Claire Negrell for the SEC analyses and Jean-Claude Roux for the scanning electronic microscopy micrographs.

\section{References}

[1] I. Raj, M. Mozetic, V. . Jayachandran, J. Jose, S. Thomas, and N. Kalarikkal, "Fracture resistant, Antibiofilm adherent, self -assembled PMMA/ZnO nanoformulations for Biomedical applications: Physico-chemical and biological perspectives of nano reinforcement," Nanotechnology, vol. 29, no. 30, 2018.

[2] R. Aradhana, S. Mohanty, and S. K. Nayak, "Comparison of mechanical, electrical and thermal properties in graphene oxide and reduced graphene oxide filled epoxy nanocomposite adhesives," Polymer (Guildf)., vol. 141, pp. 109-123, Apr. 2018.

[3] A. Lazzeri, S. M. Zebarjad, M. Pracella, K. Cavalier, and R. Rosa, "Filler toughening of plastics. Part 1 - The effect of surface interactions on physicomechanical properties and rheological behaviour of ultrafine CaCO3/HDPE nanocomposites," Polymer (Guildf)., vol. 46, no. 3, pp. 827-844, 2005.

[4] F. Laraba-Abbes, P. lenny, and R. Piques, "A new 'tailor-made' methodology for the mechanical behaviour analysis of rubber-like materials: 1 . Kinematics 
measurements using a digital speckle extensometry," Polymer (Guildf)., vol. 44, no. 3, pp. 807-820, 2003.

[5] M. P. Foulc, A. Bergeret, L. Ferry, P. lenny, and A. Crespy, "Study of hygrothermal ageing of glass fibre reinforced PET composites," Polym. Degrad. Stab., vol. 89, no. 3, pp. 461-470, 2005.

[6] B. K. Satapathy, R. Weidisch, P. Pötschke, and A. Janke, "Tough-to-brittle transition in multiwalled carbon nanotube (MWNT)/polycarbonate nanocomposites," Compos. Sci. Technol., vol. 67, no. 5, pp. 867-879, 2007.

[7] E. J. Kramer and L. L. Berger, "Fundamental Processes of Craze Growth and Fracture," Adv. Polym. Sci., pp. 1-68, 1990.

[8] A. Perthué et al., "Impact of particle size in PE/ATH composites: The relationship between the interphase and water uptake," Prog. Org. Coatings, vol. 114, no. October 2017, pp. 145-153, 2018.

[9] E. Reynaud, T. Jouen, C. Gauthier, G. Vigier, and J. Varlet, "Nanofillers in polymeric matrix: A study on silica reinforced PA6," Polymer (Guildf)., vol. 42, no. 21, pp. 8759-8768, 2001.

[10] Y. Zare and H. Garmabi, "Thickness, modulus and strength of interphase in clay/polymer nanocomposites," Appl. Clay Sci., vol. 105-106, pp. 66-70, 2015.

[11] B. J. Ash, R. W. Siegel, and L. S. Schadler, "Glass-transition temperature behavior of alumina/PMMA nanocomposites," J. Polym. Sci. Part B Polym. Phys., vol. 42, no. 23, pp. 4371-4383, 2004.

[12] Y. Joliff, W. Rekik, L. Belec, and J. F. Chailan, "Study of the moisture/stress effects on glass fibre/epoxy composite and the impact of the interphase area," Compos. Struct., vol. 108, no. 1, pp. 876-885, 2014.

[13] I. Sevostianov and M. Kachanov, "Effect of interphase layers on the overall elastic and conductive properties of matrix composites. Applications to nanosize inclusion," Int. J. Solids Struct., vol. 44, no. 3-4, pp. 1304-1315, 2007.

[14] P. Rittigstein, R. D. Priestley, L. J. Broadbelt, and J. M. Torkelson, "Model polymer nanocomposites provide an understanding of confinement effects in real nanocomposites," Nat. Mater., vol. 6, no. 4, pp. 278-282, 2007.

[15] D. Pitsa and M. G. Danikas, "Interfaces Features in Polymer Nanocomposites: a Review of Proposed Models," Nano, vol. 06, no. 06, pp. 497-508, 2011.

[16] D. Ciprai, K. Jacob, and R. Tannenbaum, "Characterization of polymer 
nanocomposite interphase and its impact on mechanical properties," Macromolecules, vol. 39, no. 19, pp. 6565-6573, 2006.

[17] Z. S. Petrovic, I. Javni, A. Waddon, and G. Banhegyi, "Structure and Properties of Polyurethane - Silica," J. Appl. Polym. Sci., vol. 76, pp. 133-151, 1999.

[18] C. M. Hadden, B. D. Jensen, A. Bandyopadhyay, G. M. Odegard, A. Koo, and R. Liang, "Molecular modeling of EPON-862/graphite composites: Interfacial characteristics for multiple crosslink densities," Compos. Sci. Technol., vol. 76, pp. 92-99, 2013.

[19] A. Bansal et al., "Quantitative equivalence between polymer nanocomposites and thin polymer films," Nat. Mater., vol. 4, no. 9, pp. 693-698, 2005.

[20] B. Kim, J. Choi, S. Yang, S. Yu, and M. Cho, "Multiscale modeling of interphase in crosslinked epoxy nanocomposites," Compos. Part B Eng., vol. 120, pp. 128-142, 2017.

[21] T. Kang, J. H. Lee, and S.-G. Oh, "Dispersion of surface-modified silica nanoparticles in polyamide-imide (PAI) films for enhanced mechanical and thermal properties," J. Ind. Eng. Chem., vol. 46, pp. 289-297, 2017.

[22] J. K. Kim, M. L. Sham, and J. Wu, "Nanoscale characterization of interphase in silane treated glass fibre composites," Compos. Part A Appl. Sci. Manuf., vol. 32, no. 5, pp. 607-618, 2001.

[23] R. Garcia and R. Proksch, "Nanomechanical mapping of soft matter by bimodal force microscopy," Eur. Polym. J., vol. 49, no. 8, pp. 1897-1906, 2013.

[24] A. Labuda, M. Kocun, W. Meinhold, D. Walters, and R. Proksch, "Generalized Hertz model for bimodal nanomechanical mapping," Beilstein J. Nanotechnol., vol. 7, no. 1, pp. 970-982, 2016.

[25] Y. He, Y. Yan, Y. Geng, and Z. Hu, "Fabrication of none-ridge nanogrooves with large-radius probe on PMMA thin-film using AFM tip-based dynamic plowing lithography approach," J. Manuf. Process., vol. 29, pp. 204-210, 2017.

[26] E. H. Barhdadi, P. Lipinski, and M. Cherkaoui, "Four phase model: A new formulation to predict the effective elastic moduli of composites," J. Eng. Mater. Technol. Trans. ASME, vol. 129, no. 2, pp. 313-320, 2007.

[27] E. Herve and A. Zaoui, "n-Layered inclusion-based micromechanical modelling," Int. J. Eng. Sci., vol. 31, no. 1, pp. 1-10, 1993.

[28] Y. Zare and K. Y. Rhee, "Development of Hashin-Shtrikman model to determine the roles and properties of interphases in clay/CaCO3/PP ternary 
nanocomposite," Appl. Clay Sci., vol. 137, pp. 176-182, 2017.

[29] T. Parpaite, B. Otazaghine, A. Taguet, R. Sonnier, A. S. Caro, and J. M. LopezCuesta, "Incorporation of modified Stöber silica nanoparticles in polystyrene/polyamide- 6 blends: Coalescence inhibition and modification of the thermal degradation via controlled dispersion at the interface," Polym. (United Kingdom), vol. 55, no. 11, 2014.

[30] T. Parpaite, B. Otazaghine, A. S. Caro, A. Taguet, R. Sonnier, and J. M. LopezCuesta, "Janus hybrid silica/polymer nanoparticles as effective compatibilizing agents for polystyrene/polyamide-6 melted blends," Polym. (United Kingdom), vol. 90, 2016.

[31] K. S. Rao, K. El-Hami, T. Kodaki, K. Matsushige, and K. Makino, "A novel method for synthesis of silica nanoparticles," J. Colloid Interface Sci., vol. 289, no. 1, pp. 125-131, 2005.

[32] L. Chen, Z. Xu, H. Dai, and S. Zhang, "Facile synthesis and magnetic properties of monodisperse Fe3O4/silica nanocomposite microspheres with embedded structures via a direct solution-based route," J. Alloys Compd., vol. 497, no. 1-2, pp. 221-227, 2010.

[33] A. K. Nozawa, H. Gailhanou, L. Raison, P. Panizza, H. Ushiki, E. Sellier, J. P. Delville and M. H. Delville, "Smart Control of Monodisperse Stöber Silica Particles: Effect of Reactant Addition Rate on Growth Process," Langmuir, vol. 21, pp. 1516-1523, 2005.

[34] A. Siot et al., "Correlation between process and silica dispersion/distribution into composite: Impact on mechanical properties and Weibull statistical analysis," Polym. Test., vol. 70, no. April, pp. 92-101, 2018.

[35] J. Honerkamp and J. Weese, "A nonlinear regularization method for the calculation of relaxation spectra," Rheol. Acta, vol. 32, no. 1, pp. 65-73, 1993.

[36] M. Feldmann, H.-P. Heim, and J.-C. Zarges, "Influence of the process parameters on the mechanical properties of engineering biocomposites using a twin-screw extruder," Compos. Part A Appl. Sci. Manuf., vol. 83, pp. 113-119, 2016.

[37] M. Feldmann, "The effects of the injection moulding temperature on the mechanical properties and morphology of polypropylene man-made cellulose fibre composites," Compos. Part A Appl. Sci. Manuf., vol. 87, pp. 146-152, 2016. 
[38] L. Banks-Sills, D. G. Shiber, V. Fourman, R. Eliasi, and A. Shlayer, "Experimental determination of mechanical properties of PMMA reinforced with functionalized CNTs," Compos. Part B Eng., vol. 95, pp. 335-345, 2016.

[39] M. L. Saladino, T. E. Motaung, A. S. Luyt, A. Spinella, G. Nasillo, and E. Caponetti, "The effect of silica nanoparticles on the morphology, mechanical properties and thermal degradation kinetics of PMMA," Polym. Degrad. Stab., vol. 97, no. 3, pp. 452-459, 2012.

[40] A. Tessema et al., "Effect of filler loading, geometry, dispersion and temperature on thermal conductivity of polymer nanocomposites," Polym. Test., vol. 57, pp. 101-106, 2017.

[41] A. A. Cuadri and J. E. Martín-Alfonso, "Thermal, thermo-oxidative and thermomechanical degradation of PLA: A comparative study based on rheological, chemical and thermal properties," Polym. Degrad. Stab., vol. 150, no. January, pp. 37-45, 2018.

[42] J. Besson, R. Leriche, R. Foerch, and G. Cailletaud, "Object-Oriented Programming Applied to the Finite Element Method Part II. Application to Material Behaviors," Rev. Eur. des Éléments Finis, 2012.

[43] T. Adachi, W. Araki, and M. Higuchi, "Mixture law including particle-size effect on fracture toughness of nano- and micro-spherical particle-filled composites," Acta Mech., vol. 214, no. 1-2, pp. 61-69, 2010.

[44] Y. Zare, "RSC Advances Assumption of interphase properties in classical Christensen - Lo model for Young's modulus of polymer nanocomposites reinforced with spherical nanoparticles," RSC Adv., vol. 5, pp. 95532-95538, 2015.

[45] M. Cherkaoui, H. Sabar, and M. Berveiller, "Micromechanical Approach of the coated inclusion problem and applications to composite materials," J. Eng. Mater. Technol., vol. 116, no. July 1994, pp. 274-278, 1994.

[46] Y. Joliff, W. Rekik, L. Belec, and J. F. Chailan, "Study of the moisture/stress effects on glass fibre/epoxy composite and the impact of the interphase area," Compos. Struct., vol. 108, no. 1, pp. 876-885, 2014.

[47] G. Alfano and M. A. Crisfield, "Finite element interface models for the delamination analysis of laminated composites: Mechanical and computational issues," Int. J. Numer. Methods Eng., vol. 50, no. 7, pp. 1701-1736, 2001.

[48] J. Cai et al., "Effects of molecular weight on poly( $\omega$-pentadecalactone) 
mechanical and thermal properties," Polymer (Guildf)., vol. 51, no. 5, pp. 10881099, 2010.

[49] Y. Zare, "A simple technique for determination of interphase properties in polymer nanocomposites reinforced with spherical nanoparticles," Polymer (Guildf)., vol. 72, pp. 93-97, 2015.

[50] R. D. Peng, H. W. Zhou, H. W. Wang, and L. Mishnaevsky, "Modeling of nanoreinforced polymer composites: Microstructure effect on Young's modulus," Comput. Mater. Sci., vol. 60, pp. 19-31, 2012. 